\title{
An Improved Mesh Adaption and Refinement Approach to Cavitation Simulation (MARCS) of Propellers
}

\author{
Naz Yilmaz $^{1 *}$, Mehmet Atlar ${ }^{1}$, Mahdi Khorasanchi ${ }^{2}$ \\ ${ }^{1}$ Naval Architecture, Ocean and Marine Engineering, University of Strathclyde, Glasgow, \\ United Kingdom \\ ${ }^{2}$ Department of Mechanical Engineering, Sharif University of Technology, Tehran, Iran \\ (formerly with Naval Architecture, Ocean and Marine Engineering, University of \\ Strathclyde, Glasgow, United Kingdom)
}

Corresponding Author:

*Naz Yilmaz, naz.gorener@strath.ac.uk

Naval Architecture, Ocean and Marine Engineering Department

University of Strathclyde,

100 Montrose Street, Glasgow

United Kingdom, G4 0LZ 


\section{Abstract}

This paper presents the improvements of cavitation modelling for marine propellers particularly developing tip vortex cavitation. The main purpose of the study is to devise a new approach for modelling tip vortex cavitation using Computational Fluid Dynamics (CFD) methods with commercial software, STAR-CCM+. The INSEAN E779A model propeller was used for this study as a benchmark propeller. Utilizing this propeller, firstly, validation studies were conducted in non-cavitating conditions together with grid and time step uncertainty studies. Then, the cavitation was simulated on the propeller using a numerical cavitation model, which is known as the Schnerr-Sauer model, based on the Rayleigh-Plesset equation. While a Reynolds Averaged Navier Stokes (RANS) model was used for open water simulations, Detached Eddy Simulations (DES) and Large Eddy Simulations (LES) models were preferred for cavitation simulations to capture the cavitation and evaluate its effect on propeller performance accurately. Although the comparison with the benchmark experimental data showed good agreement for the thrust and torque coefficients as well as sheet cavitation pattern, tip vortex cavitation could not be adequately simulated using the existing method. After an evaluation of the interaction between cavitation modelling and generated meshes, two techniques, which involved volumetric control and adaptive mesh refinement, were used in combination on the region where the tip vortex cavitation is likely to occur. The first technique, which is called a 'volumetric control method', was developed using spiral geometry around the propeller tip region to generate a finer mesh for capturing tip vortex cavitation. Although this method gave better tip vortex cavitation extension than the method without any mesh refinement or with tube refinement, it still required to be improved to extend the tip vortices further into the propeller slipstream. The second method, which is called 'adaptive mesh refinement', was introduced using the pressure distribution data from the results of the 'volumetric control method'. This improved approach, which is called "Mesh Adaption and Refinement for Cavitation Simulation (MARCS)", has been successfully applied to simulate the tip vortices trailing from the blades of the INSEAN E779A propeller as demonstrated in the paper. The results of the simulations showed an excellent agreement with the experiments in the open literature by tracking the tip vortex cavitation along this propeller's slipstream.

\section{Keywords}

Propeller Cavitation, Tip vortex cavitation, CFD, DES, LES 


\section{INTRODUCTION}

Cavitation is a complex phenomenon which can affect the propeller efficiency and create radiated noise, erosion and vibration. There are different types of cavitation (e.g. sheet, bubble, tip vortex, etc.), and each type of cavitation affects the propeller performance differently. While some sheet cavitation is associated with erosive effects on propeller blade surfaces, tip vortex cavitation is associated with radiated noise, particularly for naval, survey and cruise ships. The requirements for efficient propeller design and higher ship speed and power mean that avoiding cavitation is not possible, but must often be managed under challenging circumstances.

In the past 40 years, studies of propeller cavitation have focused on sheet cavitation largely, as it affected propeller hull excited vibration. Thus, the numerical methods such as lifting surface and Boundary Element Methods (BEM) have been developed and used to predict propeller performance including sheet cavitation. Lee (1979) developed a numerical lifting surface procedure to predict both the steady and unsteady performance of sub-cavitating propellers. Due to the mainly more accurate representation of the three-dimensional effect, BEM was implemented by Fine and Kinnas (1993) for the analysis of the flow around 3-D cavitating hydrofoils. Kinnas and Hsin (1992) analysed unsteady flow around a marine propeller using a potential-based low-order BEM. Afterwards, cavity shapes were validated using the same method by Kinnas and Fine (1994) for a cavitating marine propeller. In later years, Young and Kinnas (2001) analysed sheet cavitation on a propeller using BEM and predicted forces and cavity shapes that showed good agreement with experimental results. BEM was implemented in the PROCAL code by Vaz and Bosschers (2006) and applied to the benchmark DTMB P4119 and Seiun-Maru propeller in non-cavitating conditions, for the MARIN S-propeller and the INSEAN E779A propellers in cavitating conditions.

Nowadays it is accepted that sheet cavitation for marine propellers can be predicted reasonably accurately with existing methods such as lifting surface, BEM and even more accurately with computational fluid dynamics methods (CFD) due to better modelling of the physics of the flow, thanks to developing computational power and technology. However, simulating the tip vortex cavitation of a propeller is still challenging for propeller cavitation research. Fine (1992) mentioned that the prediction of sheet cavitation using BEM sometimes gives unstable convergence due to the lack of tip vortex cavitation modelling. On the other hand, Lee (2002) applied a low order potential-based BEM in his PhD dissertation 
for not only sheet cavitation but also tip vortex cavitation. Lee and Kinnas (2004) investigated tip vortex cavitation for a two-dimensional tip vortex, three-dimensional hydrofoil and a marine propeller using BEM and concluded that convergence studies and comparisons were satisfactory for the foils while the method required further development for marine propellers.

In the past decade, CFD methods have become common, superseding potential flow based BEM methods, with RANS primarily being preferred by researchers due to computational time and costs. Hsiao and Pauley (1998) used incompressible RANS computations with the Baldwin-Barth turbulence model for simulating tip vortex cavitation. They also tested a modified tip propeller which delayed cavitation inception without reducing propeller performance. Tip vortex cavitation inception was simulated for a marine propeller using RANS including scaling effects by Hsiao and Chahine (2008). Lately, a RANS method with the Schnerr-Sauer cavitation model was implemented by Gaggero et al. (2014) for simulating tip vortex cavitation for two ducted propellers. Although RANS was proved to be a reliable tool for the prediction of sheet cavitation, further studies - especially for tip vortex cavitationare still required (Gaggero et al., 2014). In particular, tip vortex extent can be highly sensitive to the choice of the RANS turbulence model in non-cavitating (Guilmineau et al., 2015) and cavitating flows (Viitanen \& Siikonen, 2017).

In contrast to the RANS model, scale-resolving simulations can model small-scale motions and resolve the large scales of turbulence. For scale-resolving simulations, there are two approaches, namely DES and LES models, which are available in one of the widely used commercial CFD codes for marine applications, known as STAR-CCM+ (STAR-CCM+ User Guide, 2018). DES and LES models have thus been preferred more commonly for simulating complex physical phenomena such as cavitation. In the present study, after making comparisons between RANS, DES and LES models for propeller performance characteristics $\left(\mathrm{K}_{\mathrm{T}}, \mathrm{K}_{\mathrm{Q}}\right.$ and $\left.\eta_{0}\right)$ and cavitation patterns, the LES model was preferred for simulating particularly tip vortex cavitation.

The main objective of the study presented in this paper is to develop a new meshing technique to simulate tip vortex cavitation of a propeller and to track its extension in the propeller's slipstream. This work is a precursor to the study of full propeller-hull-rudder interaction and has been achieved using CFD methods through an investigation of the interaction between tip vortex cavitation modelling and generated meshes. For this purpose, 
the commercial CFD code STAR-CCM+, which implements the Schnerr-Sauer cavitation model for simulating cavitation, has been used, Schnerr and Sauer (2001), (STAR-CCM+ User Guide, 2018). Detailed mesh generation techniques have been investigated for capturing cavitating bubbles, bubble dynamics, cavitation inception and bubble radius. Although each bubble seed cannot be modelled and tracked for its motion in space using the cavitation models in STAR-CCM+, these phenomena have been investigated profoundly through wise use of the software and mesh generation capabilities.

The proposed new meshing technique is based on the relationship between the generated mesh size and bubble radius. If the generated mesh has a larger size than required, tip vortex cavitation cannot be simulated. Hence studies related to cavitating bubble radius were investigated in the literature. Within this framework, e.g. Kuiper (1981) performed cavitation tests using a model scale propeller (Propeller $\mathrm{V}$ ) at advance coefficient, $\mathrm{J}=0.3,0.4$ and 0.5 and focused on tip vortex cavitation. During the tests, the radii of the cavitating core corresponding to various cavitation index values were measured, and a relationship between the cavitation index and the core radius was established. Based on this kind of study, the relation between bubble radius, mesh size and simulating tip vortex cavitation is propounded in this study.

Additionally, the benchmark propeller (INSEAN E779A), which was used in the Rome Workshop in the VIRTUE Project, was preferred in this study to validate the technique to be developed. This model propeller, which is a four-bladed FPP (Fixed Pitch Propeller) with small skew, was designed in 1959 and was tested by INSEAN (Instituto Nazionale di Studi ed Esperienze di Architettura Navale) in non-cavitating and cavitating conditions. The results of the Rome Workshop on the E779A propeller, including cavitation, were presented by Salvatore et al. (2009). Different computational models, i.e. RANS, LES and BEM were used for comparison in non-cavitating and cavitating conditions for the propeller performance including pressure distributions and cavitation patterns for the latter condition on the blades. A theoretical and experimental study was conducted in uniform flow for cavitation measurement of the E779A propeller by Pereira et al. (2004). Another experimental study was carried out in a cavitation tunnel in non-uniform flow by Pereira et al. (2016) for describing a correlation between cavitation patterns on blades and near-field pressures. Besides these experimental studies, the E779A propeller had been the subject of numerous simulations studies by using CFD methods and comparisons with the experimental results as 
a benchmark propeller. For example, Morgut and Nobile performed CFD simulations using the three mass transfer models of Zwart, Full Cavitation Model (FCM) and Kunz for the E779A as well as for the Potsdam Propeller Test Case (PPTC) including inclined shaft conditions (Morgut and Nobile, 2012). Vaz et al. (2015) used RANS and RANS-BEM coupled approaches in non-cavitating and cavitating conditions to predict propeller performance, pressure distributions and cavitation volume for the latter condition. Bensow and Bark also simulated E779A propeller in cavitating conditions to predict cavity extent on the blade surface as well as tip vortex development and its interaction with the sheet cavity using an LES model in OpenFOAM (Bensow and Bark, 2010). Guilmineau et al. (2015) discussed various turbulence closures such as RANS SST k- $\omega$, Algebraic Reynolds Stress Model (EARSM) and DES k- $\omega$ model to predict the wake of the E779A propeller. Although the instabilities of the wake cannot be predicted with RANS, the evaluation of the tip vortices and the prediction of the instabilities of the wake can be achieved using a DES model. Although these simulations succeed in validating propeller performance and cavitation patterns on the blade surface of the E779A propeller, tip vortex cavitation could not be simulated especially including its extension into the propeller's slipstream.

In a recent international symposium on propellers (SMP'17) the authors' pilot study using the STAR-CCM+ code which has been the building block of the study presented in this paper, Yilmaz et al. (2017). In this pilot study, a tube geometry was created around the propeller tip area and computations were concentrated in this tube for extending the tip vortex cavitation into the propeller's slipstream effectively. Yilmaz et al. presented the preliminary results for a limited range of tip vortex extensions on two benchmark propellers (PPTC inclined shaft case and INSEAN E779A). Since this reporting, the authors have further developed this method and applied it to the E779A propeller to achieve a greater extension of the tip vortex development in the propeller's slipstream, as presented in the remaining part of this paper. It is interesting to report from the same international symposium that, with a similar mesh refinement approach and using an adaptive mesh technique, Lloyd et al. (2017) also presented the results of their cavitation simulations using the in-house CFD software, ReFRESCO, although this was for only one blade. Windt and Bosschers have also applied an adaptive mesh refinement approach using the jump based estimator with ReFRESCO for a wing and a propeller. Although adaptive mesh refinement improves the accuracy of the calculation for the wing, good results could not be obtained from this application for the propeller, which still needs to be investigated (Windt \& Bosschers, 2015). 
Viitanen and Siikonen have also simulated a single blade of a model-scale marine propeller in cavitating conditions with a compressible homogenous flow model and a novel method for solving phase volume fractions (Viitanen \& Siikonen, 2017). The results showed good agreement regarding not only the propeller performance coefficient and local flow phenomena but also tip vortex cavitation extension in the propeller slipstream. Budich et al. (2015) applied a mesh alignment technique to improve the accuracy of the propeller wake structure for capturing tip vortex cavitation including compressible shock wave dynamics. This method has also helped to investigate surface loads due to cavity dynamics, collapse phenomena and its effects on erosion risks. Phillips and Turnock have also applied an improved version of the Vortfind algorithm increasing the mesh resolution to have sufficient mesh density for capturing vortex cores to investigate propeller rudder and hull interaction (Phillips \& Turnock, 2011). However, this study is not for cavitating vortex flow.

Despite such recent studies the full simulation of tip vortex cavitation and prediction of propeller performance of cavitating propellers, are not yet satisfactory. The present study, therefore, aims to contribute to extending the tip vortex cavitation model accurately in the propeller slipstream using a commercial code, but with a new, efficient meshing approach.

Within the framework of the above introduction and primary objective, the paper continues with the presentation of the theoretical and numerical model used in the CFD code at $\$ 2$. A summary of the pilot study and associated results, which provided the basis for the approach presented in this paper, are given in $\S 3$. As required for any CFD study, the validation and verification of the method using a formal procedure are presented in $\S 4$ for non-cavitating simulations with the benchmark propeller while the details of the new mesh refinement techniques capturing tip vortex cavitation for the same propeller are presented in $\S 5$. An overall summary of the new approach including a flowchart for simulating tip vortex cavitation is given in $\S 6$ while the associated results and discussions are included in $\S 7$. The paper ends with the concluding remarks given in $\S 8$.

\section{THEORETICAL AND NUMERICAL MODELS}

The simulations were conducted in STAR-CCM+ for non-cavitating and cavitating conditions. RANS, DES and LES for non-cavitating and cavitating conditions were preferred respectively. To capture the rotation of the propeller, a sliding mesh method was used. The mass and continuum conservation equations are given below (Ferziger \& Peric, 2002). 


$$
\begin{gathered}
\frac{\partial \rho}{\partial t}+\operatorname{div}(\rho v)=0 \\
\frac{\partial \rho}{\partial t}+\frac{\partial\left(\rho u_{i}\right)}{\partial x_{i}}=\frac{\partial \rho}{\partial t}+\frac{\partial\left(\rho u_{x}\right)}{\partial x}+\frac{\partial\left(\rho u_{y}\right)}{\partial y}+\frac{\partial\left(\rho u_{z}\right)}{\partial z}=0 \\
\frac{\partial\left(\rho u_{i}\right)}{\partial t}+\frac{\partial \rho\left(u_{j} u_{i}\right)}{\partial x_{j}}=\frac{\partial \rho\left(\tau_{i j}\right)}{\partial x_{j}}-\frac{\partial p}{\partial x_{i}}+\rho g_{i}
\end{gathered}
$$

where $\mathrm{x}, \mathrm{y}$ and $\mathrm{z}$ are the Cartesian coordinates and $u_{x}, u_{y}$ and $u_{z}$ are the Cartesian components of the velocity, $\rho$ is density, $\tau_{i j}$ is viscous part of the stress tensor and where $g$ is the component of the gravitational acceleration in the direction of the Cartesian coordinate $x_{i}$.

For turbulence modelling, the Shear Stress Transport (SST) k-omega model has been preferred in the RANS solver for non-cavitating simulations. In cavitating conditions, the SST k-omega DES model and the Wall-Adapting Local-Eddy Viscosity (WALE) subgridscale model were used for the DES and LES turbulence models, respectively, in cavitating conditions. The turbulence models that, have been used in these simulations, are well described in STAR-CCM+ User Guide (2018).

The first-order scheme was used for time discretization. The bounded-central differencing scheme which provides a good compromise between accuracy and robustness was used for discretizing the governing equations in space. This scheme is recommended for LES of complex turbulent flows such as cavitation in STAR-CCM+ User Guide, (2018).

For cavitation modelling, a Volume of Fluid (VOF) model, that is a simple multiphase model, was used for describing two phases; water and vapour. The basic VOF equations and VOF model are described in the user guide of STAR-CCM+, User Guide (2018).

For cavitation models, STAR-CCM+ uses the same homogeneous seed-based approach described in Muzaferija et al., (2017). Although these approaches are not able to capture all of the physical phenomena present, they are proven methods for use in academic and industrial studies. Seed-based mass transfer models are commonly used for gas dissolution and cavitation. For cavitation phenomena, two interacting phases are described as the liquid (water) and gas (vapour) transferring the mass between water and vapour (STAR-CCM+, User Guide 2018).

In the software, the Schnerr-Sauer cavitation model, which is based on the Rayleigh-Plesset equation, was used for the cavitation simulations. According to this model, the bubbles were 
accepted as spherical, and all seeds had the same radius at the beginning of the simulations. Since each bubble cannot be modelled individually, the cavitation is modelled using some bubbles in a control volume. According to this approach, the number of bubbles can be calculated at any time from:

$$
N=n_{0} a_{l} V
$$

where $V$ is volume, $a_{l}$ is volume fraction of liquid and $n_{0}$ is the number of bubbles per unit volume of liquid. The total vapour volume inside the control volume is calculated as follows.

$$
V_{v}=N V_{b}
$$

where $V_{b}$ is the volume of one bubble.

And the volume of one bubble can be calculated as follows, where $R$ is the radius of one bubble.

$$
V_{b}=\frac{4}{3} \pi R^{3}
$$

And the volume of a fraction of vapour, $a_{v}$ can be described by the following equation, including the bubble radius.

$$
a_{v}=\frac{V_{v}}{V}=\frac{N V_{b}}{V}=\frac{4}{3} \pi R^{3} n_{0} a_{l}
$$

The radius of a bubble can be calculated if the volume fraction is known from Equation 7.

While the bubbles are moving with the flow and the time is changing, the change of volume of every bubble can be computed as follows.

$$
\frac{d V_{b}}{d t}=4 \pi R^{2} \frac{d R}{d t}=4 \pi R^{2} v_{r}
$$

where $v_{r}$ is the bubble growth velocity. At this stage, to calculate the bubble growth velocity, an additional model is needed. This term can be calculated from the Schnerr-Sauer model.

Before describing the bubble growth velocity, for growth and collapse of the bubbles in a control volume, a source term for the volume fraction of vapour is given in Equation 9.

$$
Q_{V}=N \frac{d V_{b}}{d t}=4 \pi n_{0}\left(1-a_{v}\right) V R^{r} v_{r}
$$

The volume fraction of vapour can be calculated as: 


$$
\begin{aligned}
& a_{v}=\frac{V_{v}}{V}=\frac{V_{v}}{V_{v}+V_{l}} \\
& a_{v}=\frac{n_{0} \frac{4}{3} \pi R^{3}}{1+n_{0} \frac{4}{3} \pi R^{3}}
\end{aligned}
$$

and the vapour bubble radius can be computed from Equation 10 and then the seed diameter can be calculated.

Using the Scherr-Sauer model, the bubble growth rate is estimated using Equation 11.

$$
\left(\frac{d R}{d t}\right)^{2}=\frac{2}{3}\left(\frac{p_{s a t}-p_{\infty}}{\rho_{l}}\right)
$$

The cavitation number based on the rotational speed of the propeller is defined as

$$
\sigma_{\mathrm{n}}=\frac{\mathrm{p}-\mathrm{p}_{\mathrm{sat}}}{0.5 \rho_{\mathrm{l}}(\mathrm{nD})^{2}}
$$

where $p$ is the tunnel pressure, $p_{\text {sat }}$ is the vapour pressure, $\rho_{l}$ is the density of the fluid, $n$ is the rotation rate, and $D$ is the diameter of the propeller.

The magnitudes of the seed diameter and seed density chosen for this study were the default values provided in STAR-CCM+, namely, 1.0E-6 m and 1.0E12 $\mathrm{m}^{3}$ respectively. The tunnel pressure was calculated from the rotational cavitation number using Equation 12 and used as a reference pressure value in the software.

The advance ratio is defined as:

$$
\mathrm{J}=\frac{\mathrm{V}_{\mathrm{A}}}{\mathrm{nD}}
$$

where $V_{A}$ is the advance velocity of the fluid. Thrust and torque coefficient of the propeller is calculated as:

$$
\begin{aligned}
K_{T} & =\frac{T}{\rho_{l} n^{2} D^{4}} \\
K_{Q} & =\frac{Q}{\rho_{l} n^{2} D^{5}}
\end{aligned}
$$

where $T$ and $Q$ are thrust and torque values of the propeller respectively. The open water efficiency of the propeller is defined as below.

$$
\eta_{0}=\frac{J}{2 \pi} \frac{K_{T}}{K_{Q}}
$$




\section{A BRIEF REVIEW OF THE RECENT PILOT STUDY ON TIP VORTEX CAVITATION MODELLING}

As stated in Section $§ 1$, in SMP-17, the present authors have reported on a pilot study which involved an advanced mesh refinement technique by using a tube geometry around propeller's tip region for capturing tip vortex cavitation in a propeller slipstream (Yilmaz et al. 2017). This technique is the building block of the new advanced approach, which is called MARCS, presented in this paper. This section, therefore, gives a summary and results of this pilot study which enabled a limited extension of the cavitating tip vortex on the benchmark INSEAN E779A propeller which was analysed in open water and cavitating conditions. The results of this analysis were compared with previously published experimental results (Pereira et al. 2004, Salvatore et al. 2009). First, sheet cavitation was simulated successfully. Then a mesh refinement method was implemented in this analysis using the tube geometry as mentioned above and applied to initiate and extend the tip vortex cavitation in the propeller slipstream. The propeller geometry, simulation settings and brief discussion of the pilot study are summarised below while its further details can be found in Yilmaz et al. (2017).

\subsection{Propeller Geometry}

Figure 1 and Table 1 show the geometry and main particulars of the INSEAN E779A benchmark propeller, respectively.

Table 1. Particulars of the Propeller

\begin{tabular}{|l|c|}
\hline Propeller & E779A \\
\hline Number of Blades $(\mathrm{Z})$ & 4 \\
\hline Diameter $(\mathrm{D})$ & $0.227 \mathrm{~m}$ \\
\hline Pitch Ratio $(\mathrm{P} / \mathrm{D})$ & 1.1 \\
\hline Area Ratio $\left(\mathrm{A}_{\mathrm{E}} / \mathrm{A}_{0}\right)$ & 0.69 \\
\hline
\end{tabular}




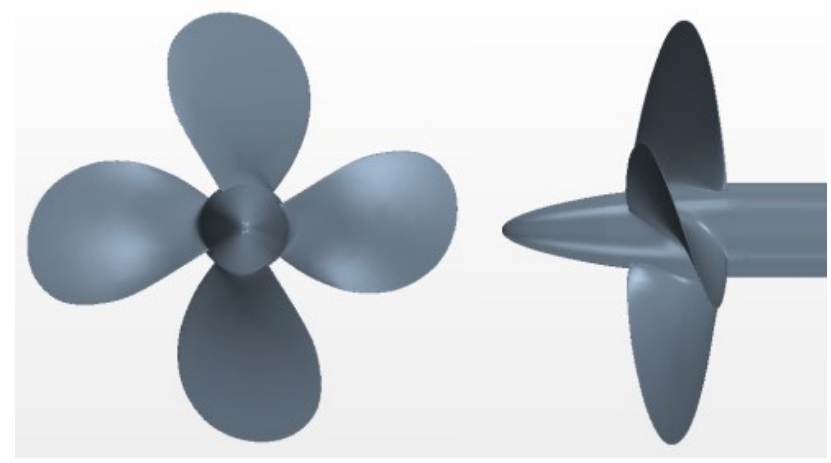

Figure 1. CAD geometry of the benchmark propeller

\subsection{Simulation Settings and Boundary Conditions}

A computational domain for the simulation of the cavitating and non-cavitating conditions of the subject propeller was prepared where the distance of the propeller centre in the axial direction from the inlet and outlet boundaries was selected as $5 D$ and $13 D$, respectively, according to the recommendation of the STAR-CCM+ user guide (2018). Computational domain geometry and boundary conditions are given in Figure 2, while Figure 3 shows the generated mesh for the cavitating simulation case. A smaller basic cell size on the surfaces of the propeller blades $(0.002 \mathrm{D}$ equal to approximately $0.5 \mathrm{~mm})$ has been used for this case compared to the non-cavitating simulations. The fine mesh number for the cavitating instance was about 14 million cells. The average $y+$ value (Figure 4) was around 1 and less for the blades and shaft respectively, using 12 prism layers and approximately $1 \mathrm{~mm}$ in total thickness. Hence, the cavitation simulations were carried out for the propeller using a DES method for two different cases in terms of $J$ and cavitation number values. The model settings of each case study are given in Table 2. Following these simulations, another cavitation case was prepared using the new tube refinement technique to simulate the cavitation including tip vortex cavitation.

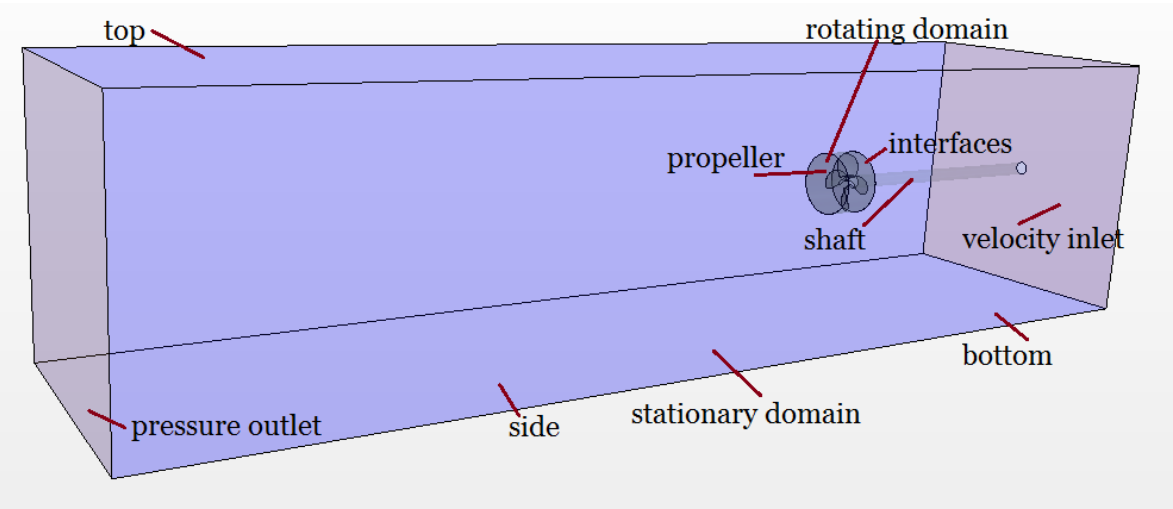

Figure 2. Computational domain and boundary conditions 


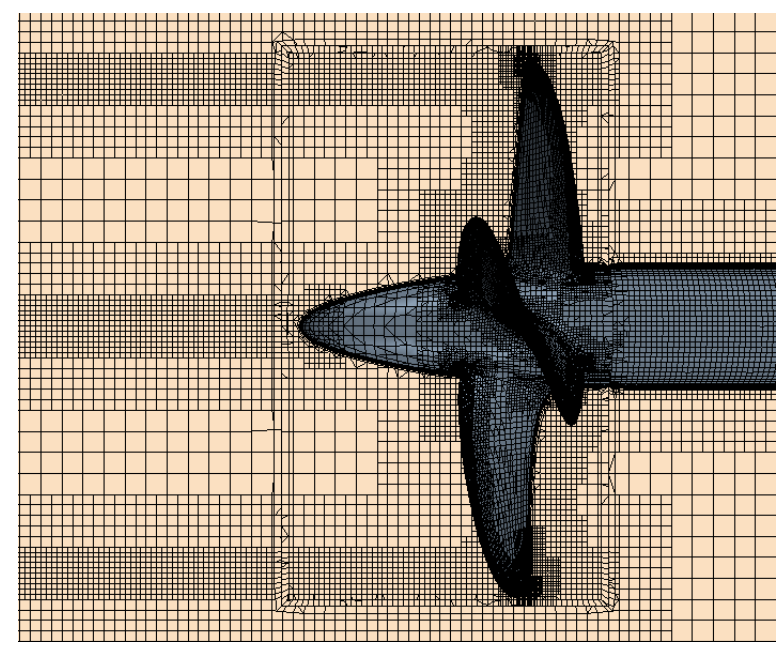

Figure 3. Computational Grid

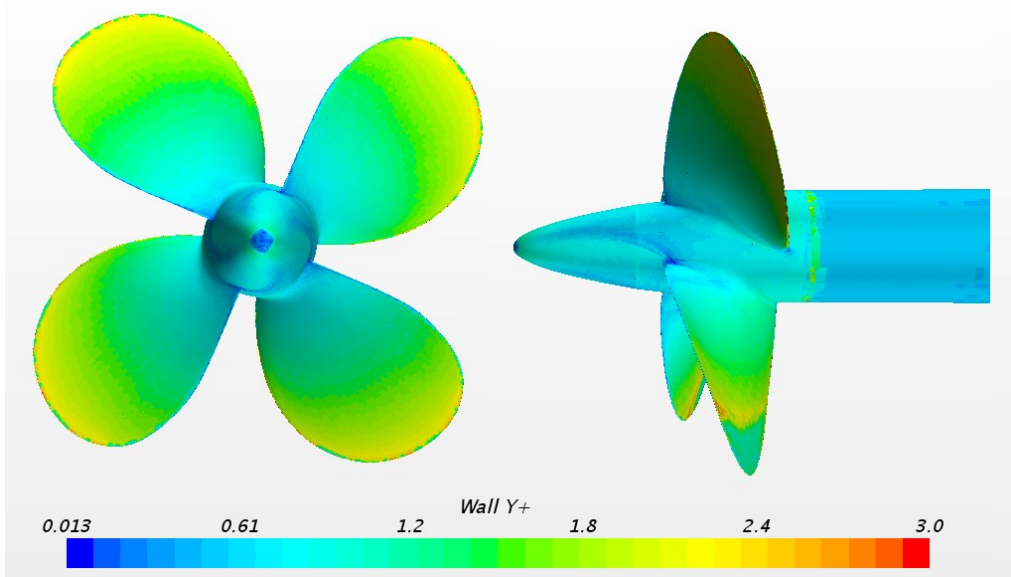

Figure 4. $y+$ on blades, hub and shaft for E779A propeller

Table 2. Model Settings for E779A propeller

\begin{tabular}{|l|c|c|c|}
\hline \multirow{2}{*}{ Variables } & \multirow{2}{*}{ Symbol } & \multicolumn{2}{c|}{ Cases } \\
\cline { 3 - 4 } & & Case 1 & Case 2 \\
\hline Advance Coefficient & $\mathrm{J}$ & 0.71 & 0.77 \\
\hline Cavitation Number based on $\mathrm{n}$ & $\sigma_{\mathrm{n}}$ & 1.763 & 2.082 \\
\hline Vapour Pressure & $\mathrm{P}_{\mathrm{v}}$ & 3170.34 & 2338 \\
\hline
\end{tabular}




\subsection{Results}

Table 3 shows the open water simulation results against experimental ones (EFD) including the simulation results from the CFD code, STAR-CCM+. Good agreement is obtained between the CFD and (EFD) results for the propeller performance coefficients.

Table 3. Open water comparison between CFD and EFD results for E779A propeller

\begin{tabular}{|c|c|c|c|c|c|c|c|}
\hline & Case & \multicolumn{3}{|c|}{$\begin{array}{c}\text { Performance } \\
\text { Coefficient }\end{array}$} & \multicolumn{3}{c|}{$\begin{array}{c}\text { Difference } \\
\text { (CFD \& EFD) }\end{array}$} \\
\hline Methods & $\mathrm{J}$ & $\mathrm{K}_{\mathrm{T}}$ & $10 \mathrm{~K}_{\mathrm{Q}}$ & $\eta_{0}$ & $\mathrm{~K}_{\mathrm{T}}$ & $10 \mathrm{~K}_{\mathrm{Q}}$ & $\eta_{0}$ \\
\hline CFD (STAR-CCM+) & 0.71 & 0.229 & 0.428 & 0.606 & $-3 \%$ & $0 \%$ & $-3 \%$ \\
\hline EFD Results & 0.71 & 0.238 & 0.429 & 0.626 & - & - & - \\
\hline
\end{tabular}

The sheet cavitation pattern simulation (Figure 5) computed for the E779A blade surfaces and hub displayed good agreement with experiment images (Pereira et al. 2004, Salvatore et al. 2009) for two different combinations of $\mathbf{J}$ and $\sigma$. However, it was concluded that this mesh and analysis method were not sufficient for capturing the extension of the cavitating tip vortex in the propeller slipstream, hence the existing method still required to be improved to simulate tip vortex cavitation by using different methods as well as the surface size and refinement of the mesh. For this reason, a helical tube was created around the propeller's tip (Figure 6) to generate a very fine mesh around the tip area where the tip vortex cavitation probably occurs. This technique provided an improvement in the tip vortex cavitation appearance (Figure 7). This improvement was directly related to the mesh size resulting from mesh refinement within the tube. Figure 7 shows that improved results were obtained for the cavitating tip vortex. However, the axial extent of the tip vortex was still under-predicted and hence needed to be improved as demonstrated in the remaining sections of this paper. 

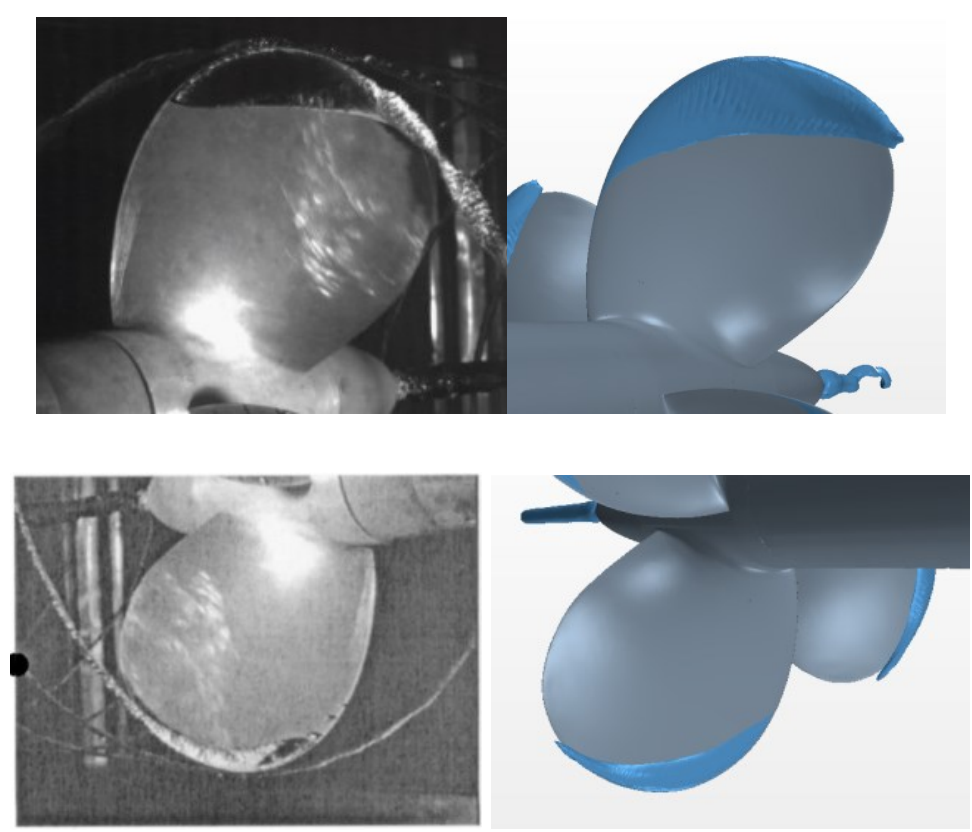

Figure 5. Comparisons between EFD and CFD, E779A propeller (Top; Case 1; J=0.71, $\sigma_{\mathrm{n}}=1.763$, Bottom; Case 2; $J=0.77, \sigma_{\mathbf{n}}=2.082$ )

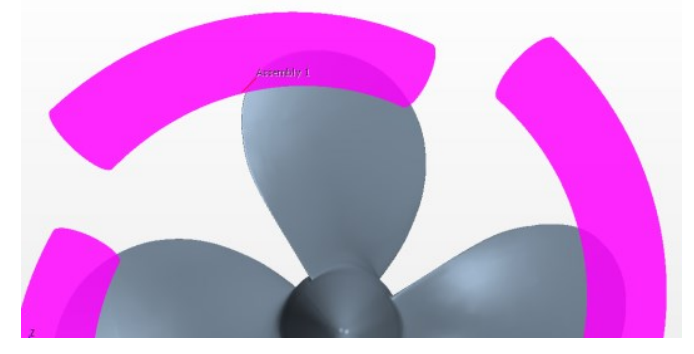

Figure 6. Helical tube around propeller's tip

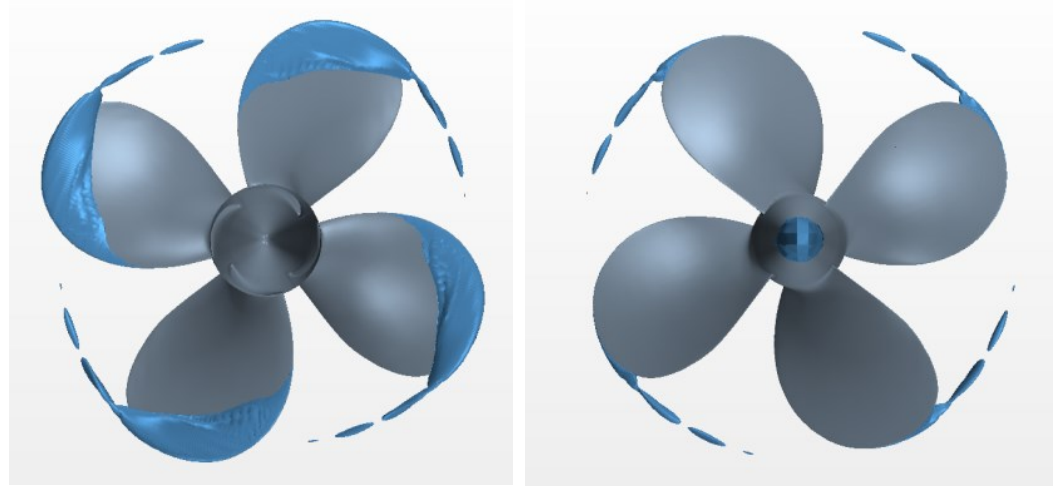

Figure 7. Tip vortex cavitation improvement using tube geometry for mesh refinement 


\section{VALIDATION AND VERIFICATION STUDIES}

Before describing further details and application of the new approach, any CFD study requires a formal procedure to be verified and validated. This is addressed in the following sections based on the methodology given in Stern et al. (2001a, 2001b).

\subsection{Methodology}

The verification and validation $(\mathrm{V} \& \mathrm{~V})$ studies were conducted for non-cavitating conditions using the two-part methodology described in Stern et al. (2001a, 2001b). This section (\$4.1), therefore, presents the methodology for the V\&V study of a CFD simulation, while Section $\S 4.2$ presents the application of the methods for RANS simulation of a propeller in open water to verify and validate the results.

The numerical simulation uncertainty includes uncertainties of the number of iterations $\left(U_{I}\right)$, the generated grid $\left(U_{G}\right)$, the time step $\left(U_{T}\right)$ and other parameters $\left(U_{P}\right)$ and can be calculated as:

$$
U_{S N}^{2}=U^{2}+U_{G}^{2}+U^{2}{ }_{T}+U_{P}^{2}
$$

To determine if the simulation has been validated, the error $(E)$ between the CFD and EFD results is compared to validation of uncertainty which is calculated as:

$$
U_{V}^{2}=U_{D}^{2}+U^{2}{ }_{S N}
$$

The error $(E)$ between the results from experiments and simulations can be calculated as follows:

$$
E=D-S
$$

where $D$ is the experimentally determined value while $S$ the value determined from the simulation.

According to this validation method, if $|E|<U_{V}$, the simulation is validated at the $U_{V}$ level. Otherwise if $\left(U_{V}<<|E|\right)$, the sign and magnitude of $E$ can be used for making improvements (Stern et al., 2001).

According to Stern's verification procedures, iterative and parametric convergence studies should be applied using multiple solutions (at least 3 ). These studies are also conducted using systematic parameters while the other parameters are kept constant. A uniform refinement ratio can be calculated as below: 


$$
r=\Delta x_{2} / \Delta x_{1}=\Delta x_{3} / \Delta x_{2}
$$

A sufficient and a good alternative for the refinement ratio may be $\sqrt{2}$ as discussed by Roache (1998). According to Roache's study, $r=2$ may be too big for industrial CFD simulations. The verification and validation studies were conducted for this paper using $r=\sqrt{ } 2$ and 2 for grid and time step convergence study, respectively, in non-cavitating conditions.

Convergence studies must be done for a minimum of three solutions to evaluate the convergence. Two solutions are not sufficient to assess the sensitivity and convergence. The type of convergence or divergence, which are defined below, and three solutions corresponding to fine $S_{1}$, medium $S_{2}$ and coarse $S_{3}$ are used.

$$
\begin{gathered}
\varepsilon_{21}=S_{2}-S_{1} \text { (Medium-Fine) } \\
\varepsilon_{32}=S_{3}-S_{2} \text { (Coarse-Medium) } \\
R=\varepsilon_{21} / \varepsilon_{32}
\end{gathered}
$$

Two different types of convergence and divergence are possible if;

(1) Monotonic Convergence: $0<R<1$

(2) Oscillatory Convergence: $R<0$

(3) Divergence: $R>1$

The errors and uncertainties can be calculated according to the type of convergence and divergence.

For monotonic convergence, a generalised Richardson Extrapolation, with correction and safety factor methods, are used for the calculation. This method is fully described by Stern et al (2001a).

For oscillatory convergence, uncertainties can be calculated as follows:

$$
U_{k}=\frac{1}{2}\left(S_{U}-S_{L}\right)
$$

where $S_{U}$ is the upper and $S_{L}$ is the lower value in the convergence history of the parameter.

For divergence, there is no method to calculate the error and uncertainties. These values cannot be estimated. 


\subsection{Open Water Simulations}

\subsubsection{Verification}

For the $\mathrm{V} \& \mathrm{~V}$ studies in non-cavitating conditions, four different grids (coarse, medium, fine and very fine) and three-time step conditions (for the fine grid) were simulated. Figure 8 shows images and Table 4 shows details of different grids for the grid independence study. In Table 4, 'Surface Size Blade' and 'Surface Size Refinement' demonstrate the surface size of the generated mesh on the propeller blade surface and the surface size of the mesh in the refinement region around propeller tip respectively. The grid convergence study was conducted at $\mathrm{J}=0.71$ using $r=\sqrt{ } 2$ with a RANS method. For the time step convergence study, three-time step conditions were prepared; namely, Coarse $(2 \Delta \mathrm{t})$, Medium $(\Delta \mathrm{t})$ and Fine $(\Delta \mathrm{t} / 2)$. The medium time step was defined as $\Delta \mathrm{t}=1.25 \times 10^{-4}$. This time step corresponds to $1.62 \mathrm{deg}$ of propeller rotation.
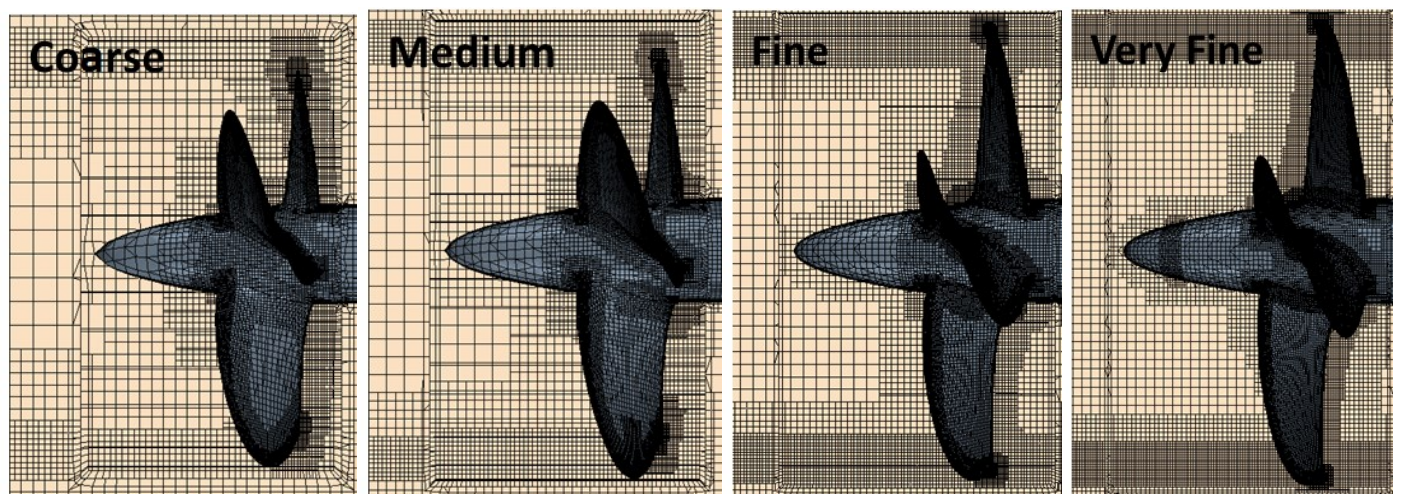

Figure 8. Different Grids for V\&V Studies

Table 4. Grid Convergence Study in Non-Cavitating Conditions

\begin{tabular}{|l|c|c|c|}
\hline $\begin{array}{l}\text { Grid } \\
\text { Convergence }\end{array}$ & $\begin{array}{c}\text { Surface Size } \\
\text { Blade }[\mathbf{m m}]\end{array}$ & $\begin{array}{c}\text { Surface Size } \\
\text { Refinement }[\mathbf{m m}]\end{array}$ & $\begin{array}{c}\text { Number of } \\
\text { Cells }\end{array}$ \\
\hline Coarse & 1.4 & 4.2 & 2022542 \\
\hline Medium & 1.0 & 3.0 & 3690159 \\
\hline Fine & 0.7 & 2.1 & 6478455 \\
\hline Very Fine & 0.5 & 1.5 & 13054684 \\
\hline
\end{tabular}

Figure 9 shows computed propeller performance coefficients for the different grids together with experimental results. As can be seen in Figure 9, the convergence types for "coarse, medium and fine" meshes are oscillatory as for "medium, fine and very fine" meshes. For 
this type of convergence, the uncertainties given in Table 6 were calculated using the upper and lower values of $\mathrm{K}_{\mathrm{T}}$ and $\mathrm{K}_{\mathrm{Q}}$ in the time history. For this time step convergence study, the propeller performance coefficients were given in Table 6 in detail. The time step uncertainty was calculated using Equation 17. (Table 6)

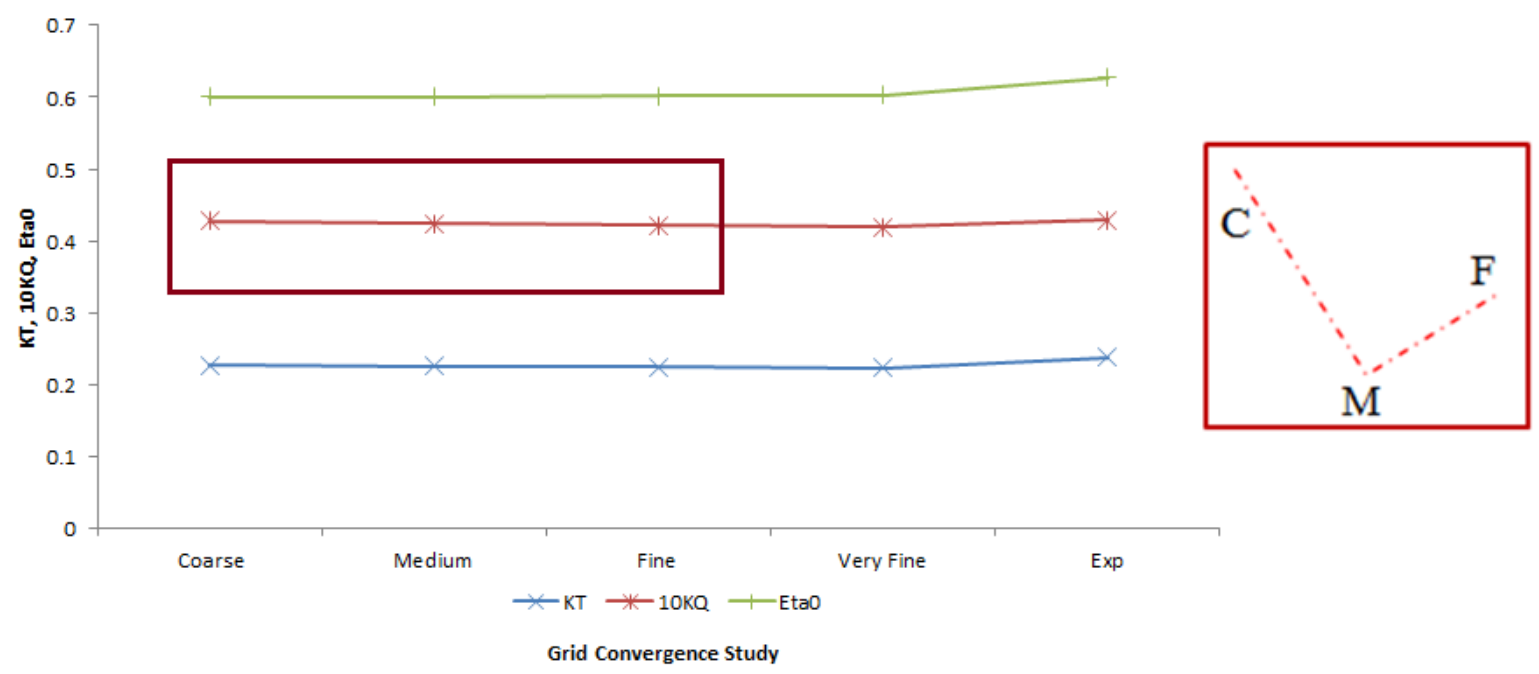

Figure 9. Grid Convergence Study

Table 5. Time Step Convergence Study in Non-Cavitating Conditions

\begin{tabular}{|c|c|c|c|c|c|}
\hline & & $\mathrm{J}$ & $\mathrm{K}_{\mathrm{T}}$ & $10 \mathrm{~K}_{\mathrm{Q}}$ & $\eta_{0}$ \\
\hline $\mathrm{Si} 3$ & $2 \Delta \mathrm{t}$ & 0.71 & 0.22910 & 0.4245 & 0.6097 \\
\hline $\mathrm{Si} 2$ & $\Delta \mathrm{t}$ & 0.71 & 0.22909 & 0.4243 & 0.6101 \\
\hline $\mathrm{Si} 1$ & $\Delta \mathrm{t} / 2$ & 0.71 & 0.22929 & 0.4244 & 0.6105 \\
\hline & $\operatorname{Exp}$ & 0.71 & 0.23800 & 0.4290 & 0.6269 \\
\hline
\end{tabular}

Table 6. Numerical Uncertainty Calculations from Grid and Time Step Convergence

\begin{tabular}{|c|c|c|}
\hline & $\mathbf{K}_{\mathbf{T}} \%$ & $\mathbf{1 0 K} \mathbf{Q} \%$ \\
\hline Grid Uncertainty (Coarse, Medium and Fine) & 0.05457 & 0.07494 \\
\hline Grid Uncertainty (Medium, Fine and Very Fine) & 0.02931 & 0.04777 \\
\hline Time Step Uncertainty & 0.17262 & 0.24710 \\
\hline
\end{tabular}




\subsubsection{Validation}

The error between CFD and EFD results $(E)$, uncertainties of validation $\left(U_{V}\right)$, experimental tests $\left(U_{D}\right)$ and numerical simulations $\left(U_{S N}\right)$ were given for $\mathrm{K}_{\mathrm{T}}$ and $\mathrm{K}_{\mathrm{Q}}$ in Table 7 and Table 8, respectively. For these calculations, Equation 17 was used, and previous data uncertainty and iterative uncertainty were neglected. The iteration errors and iterative uncertainties are accepted to be negligible compared with the grid and time step uncertainties with regards to the thrust and torque coefficient.

It is observed that the deviations between calculated and measured values in non-cavitating conditions are small, $4 \%$ for thrust and $1 \%$ for torque coefficients. Although the deviation is small between the EFD and CFD results, the uncertainty for experimental tests was assumed as $1 \%,|E|>U_{V}$ such that $K_{T}$ is not validated due to a small $U_{V}$ value for grid 1 and 2. On the other hand, $\mathrm{K}_{\mathrm{Q}}$ is validated for both grids. $\left(|\mathrm{E}|<\mathrm{U}_{\mathrm{V}}\right)$

Table 7. Validation of Thrust Coefficient $\left(K_{T}\right)$

\begin{tabular}{|c|c|c|c|c|}
\hline Grid & $\mathbf{E} \%$ & $\mathbf{U S N}_{\mathbf{S N} \%}$ & $\mathbf{U D}_{\mathbf{D}} \%$ & $\mathbf{U v} \%$ \\
\hline 1 (Coarse, Medium and Fine) & -4 & 0.180 & 1 & 1.032 \\
\hline 2 (Medium, Fine and Very Fine) & -4 & 0.175 & 1 & 1.030 \\
\hline
\end{tabular}

Table 8. Validation of Torque Coefficient $\left(K_{Q}\right)$

\begin{tabular}{|c|c|c|c|c|}
\hline Grid & $\mathbf{E \%}$ & UsN\% & UD\% & Uv\% \\
\hline 1 (Coarse, Medium and Fine) & -1 & 0.258 & 1 & 1.066 \\
\hline 2 (Medium, Fine and Very Fine) & -1 & 0.251 & 1 & 1.063 \\
\hline
\end{tabular}

Although cavitation simulations have been conducted using LES model and presented in the following sections of this paper, the propeller has been simulated in non-cavitating conditions with a RANS model for these validation and verification studies. An LES model was preferred instead of RANS to develop the more detailed mesh refinement model and capture tip vortex cavitation effectively in the propeller slipstream. Although a RANS model is sufficient to predict hydrodynamic propeller performance coefficients, it is not still satisfactory to model tip vortex cavitation phenomena as discussed in $\S 1$. Due to the switch of turbulence model from RANS to LES, another validation and verification study for LES turbulence model was considered necessary for cavitating flow conditions for this study and is presented in the following section. 


\subsection{Cavitation Simulations}

\subsubsection{Verification}

For the verification and validation studies in cavitating conditions, three different grids (coarse, medium, and fine) and three-time step conditions (for the medium grid) has been simulated following the same $\mathrm{V} \& \mathrm{~V}$ procedure presented at $\S 4.1$. While Figure 10 presents the images of different grids for the grid uncertainty studies, Table 9 demonstrates the details of refined meshes using MARCS (please see $\S 5.2$ ). V\&V studies have been conducted for cavitating conditions with an LES model which is particularly recommended by STAR$\mathrm{CCM}+$ to solve complex turbulent flows such as, tip vortex type cavitation. Three-time step conditions have been simulated for the medium grid as described in the V\&V studies for cavitating conditions using the coarse, medium and fine time step $\left(\Delta \mathrm{t} / 2=5 \times 10^{-5}\right.$ which corresponds to $0.64 \mathrm{deg}$ of propeller rotation).
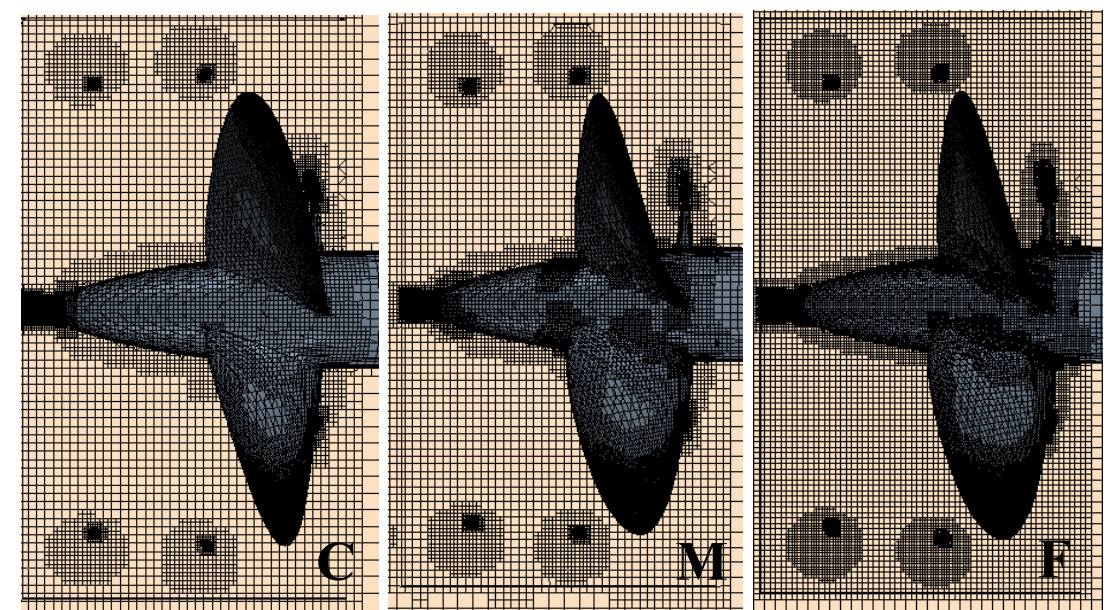

Figure 10. Different Grids for V\&V Studies (Tip Vortex Cavitation)

Table 9. Grid Convergence Study Non-Cavitating Conditions

\begin{tabular}{|l|c|c|c|c|}
\hline $\begin{array}{l}\text { Grid } \\
\text { Convergence }\end{array}$ & $\begin{array}{c}\text { Surface Size } \\
\text { Blade } \\
{[\mathrm{mm}]}\end{array}$ & $\begin{array}{c}\text { Surface Size } \\
\text { Refinement } \\
{[\mathrm{mm}]}\end{array}$ & $\begin{array}{c}\text { Refinement } \\
\text { Factor MARCS }\end{array}$ & $\begin{array}{c}\text { Number } \\
\text { of Cells } \\
{[-]}\end{array}$ \\
\hline Coarse & 1.4 & 4.2 & Cell Width / 1.5 & 8548852 \\
\hline Medium & 1.0 & 3.0 & Cell Width / 2.0 & 15702802 \\
\hline Fine & 0.7 & 2.1 & Cell Width / 3.0 & 28912661 \\
\hline
\end{tabular}


Table 10. Grid Uncertainty Study Results in Cavitating Conditions

\begin{tabular}{|c|c|c|c|}
\hline & $\mathrm{K}_{\mathrm{T}}$ & $10 \mathrm{~K}_{\mathrm{Q}}$ & $\eta_{0}$ \\
\hline Coarse & 0.2473 & 0.4593 & 0.6084 \\
\hline Medium & 0.2453 & 0.4406 & 0.6292 \\
\hline Fine & 0.2470 & 0.4435 & 0.6295 \\
\hline Exp & 0.255 & 0.460 & 0.626 \\
\hline
\end{tabular}

Table 11. Numerical Uncertainty Calculations from Grid Convergence in terms of $K_{T}, K_{Q}$ and different types of cavitation

\begin{tabular}{|c|c|c|c|c|c|}
\hline Uncertainty & $\mathbf{K}_{\mathbf{T}} \%$ & $\mathbf{1 0 K \mathbf { K } \%}$ & $\begin{array}{c}\text { Cavity } \\
\text { Volume\% } \\
\text { (Total) }\end{array}$ & $\begin{array}{c}\text { Cavity } \\
\text { Volume\% } \\
\text { (Sheet) }\end{array}$ & $\begin{array}{c}\text { Cavity } \\
\text { Volume\% } \\
\text { (Tip) }\end{array}$ \\
\hline Grid Uncertainty & 0.1345 & 0.2232 & 348.92 & 41.15 & 307.77 \\
\hline
\end{tabular}
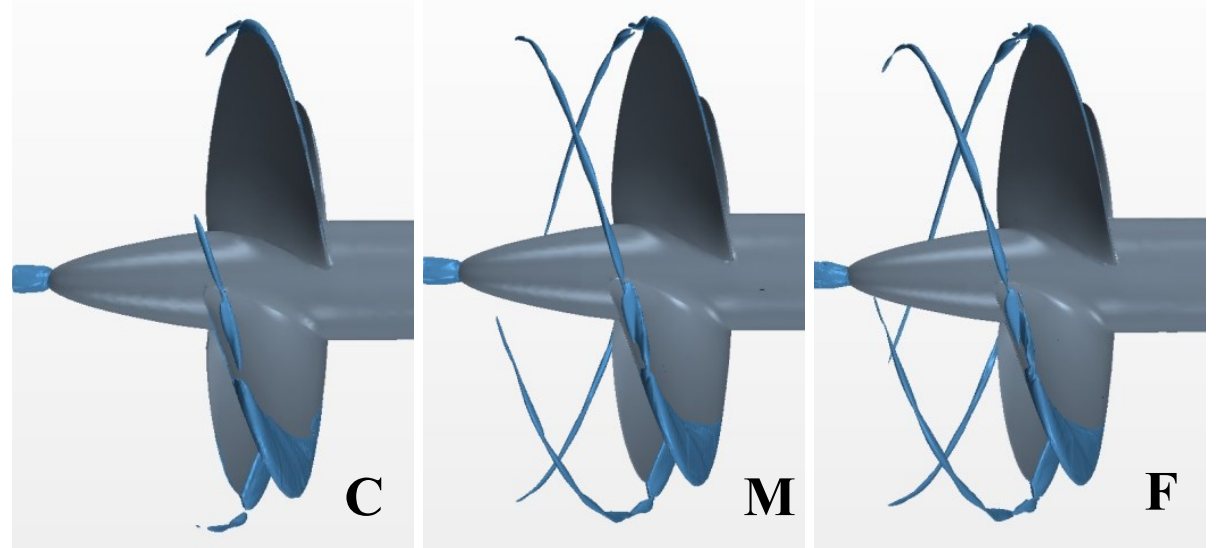

Figure 11. Tip Vortex Cavitation Extension due to different grids for V\&V studies

The results of the grid uncertanity studies have been given in Table 10 in terms of propeller hydrodynamic performance characteristics. Besides $\mathrm{K}_{\mathrm{T}}, \mathrm{K}_{\mathrm{Q}}$ and $\eta_{0}$, the numerical uncertainties have also calculated for cavitating volume with regards to different type of cavitation presented in Table 11. While Table 11 demonstrates the uncertainty calculations for cavity volume, Figure 11 shows the cavitation pattern including tip vortex cavitation extend in the propeller slipstream due to the different mesh refinements. Table 11 and Figure 11 also presents that the uncertainty values for tip vortex cavitation predictions are much higher than sheet cavitation and adaptive mesh refinement is required to solve tip vortex 
cavitation accurately. The images of the cavity extent also proves that smaller grid size (Cell Width / 3.0) is essential to be able to extend tip vortex type cavitation.

\subsubsection{Validation}

The same validation procedure has been followed with the non-cavitating simulation as described in $\S 4.2 .2$ and Table 12 has been prepared including results $(E)$, uncertainties of numerical simulations $\left(U_{S N}\right)$, experiments $\left(U_{D}\right)$ and validation $\left(U_{V}\right)$ respectively.

Table 12 shows that although the deviation is small between the EFD and CFD results (3\% for thrust and $3 \%$ for torque coefficients) the uncertainty for experimental tests in cavitating conditions was assumed to be $2 \%,|\mathrm{E}|>\mathrm{UV}_{\mathrm{V}}$ such that $\mathrm{K}_{\mathrm{T}}$ and $\mathrm{K}_{\mathrm{Q}}$ are not validated due to a small $U_{V}$ value for the grid convergence study.

Table 12. Validation of Thrust $\left(K_{T}\right)$ and Torque Coefficient $\left(K_{Q}\right)$

\begin{tabular}{|c|c|c|c|c|}
\hline & $\mathbf{E} \%$ & $\mathbf{U S N}_{\mathbf{S N}}$ & $\mathbf{U}_{\mathbf{D}} \%$ & $\mathbf{U v} \%$ \\
\hline $\mathbf{K}_{\mathbf{T}}$ & 3 & 0.1815 & 2 & 2.008 \\
\hline $\mathbf{K}_{\mathbf{Q}}$ & 3 & 0.3042 & 2 & 2.023 \\
\hline
\end{tabular}

\section{MESH REFINEMENT METHODS FOR TIP VORTEX CAVITATION}

\subsection{Volumetric Control Method (Spiral Method)}

As already reviewed in $\S 3$, the mesh refinement approach using tube geometry and generating a refined mesh around the propeller tip has not been sufficiently satisfactory to fully capture tip vortex cavitation, in particular, its extension in the slipstream. The results of the authors' pilot study confirmed the necessity to simulate tip vortex cavitation and predict propeller performance in cavitating conditions adequately. Therefore, the tube geometry was extended helicoidally from the propeller tip region all the way through the propeller slipstream. The details of this method are described below, as applied to the INSEAN propeller.

Firstly, the simulation was run with the mesh without any refinement. From this simulation, the pressure distribution behind the propeller can be obtained to create the contracting spiral geometry of the propeller slipstream in the rotating domain region. The pitch and the reduced diameter of the slipstream were calculated from the results of this first run. Figure 12 shows the spiral geometry which is starting from the propeller tip region through the propeller's 
slipstream. This geometry was used as a "volumetric control" to generate a more refined mesh in the region where the cavitation bubbles are moving with the flow and where the tip vortex cavitation may occur. A surface mesh size of $0.002 \mathrm{D}$ - the smallest possible regarding the computational power- was used in the spiral region. A finer mesh of approximately 19 million cells was generated for the cavitation case. In the previous pilot study, although RANS and DES models were used for non-cavitating and cavitating simulations respectively, LES was preferred for this study. While the mesh refinement methods extended the cavitation pattern, particularly tip vortex cavitation, the LES model also improved the results regarding the propeller performance characteristics $\left(\mathrm{K}_{\mathrm{T}}, \mathrm{K}_{\mathrm{Q}}\right.$ and $\left.\eta_{0}\right)$. The propeller performance coefficients were calculated using thrust and torque values obtained from the simulations after the solution time corresponded to at least four revolutions of the propeller.

For cavitation simulations, selected time step of $\Delta \mathrm{t}=5 \times 10^{-5} \mathrm{~s}$ corresponds to $0.648 \mathrm{deg}$ of propeller rotation. The time step is calculated such that the propeller rotates between 0.5 and 2 degrees per time step according to International Towing Tank Conference (ITTC) recommendation (ITTC, 2014).

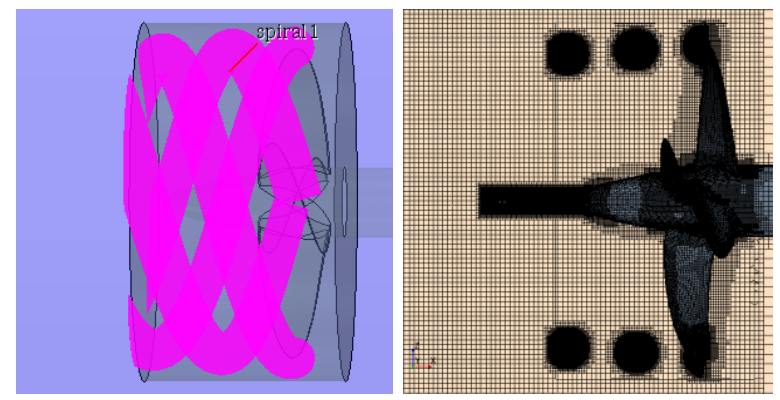

Figure 12. Left; spiral geometry in rotating domain, Right; mesh refinement due to spiral geometry

Figure 13 compares the cavitation pattern from the refined spiral mesh method with the test (EFD) images from Salvatore et al. (2009). In comparison with the previous simulations (Figure 5 and 7) of the pilot study, the new spiral mesh gives significantly better results for the cavitating slipstream extension. The cavitation pattern was created using an iso-value of 0.1 for the volume fraction of vapour. 
Solution Time 0.0667 (s)

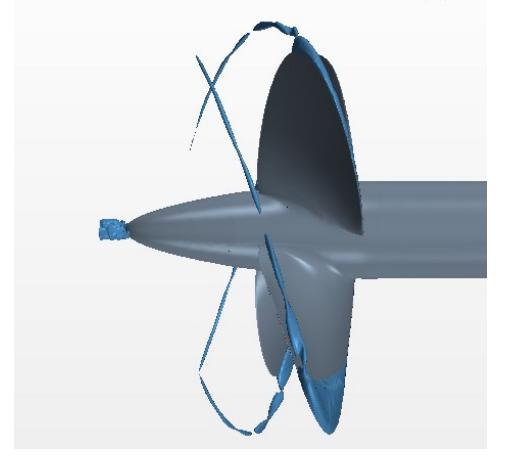

Solution Time 0.0667 (s)

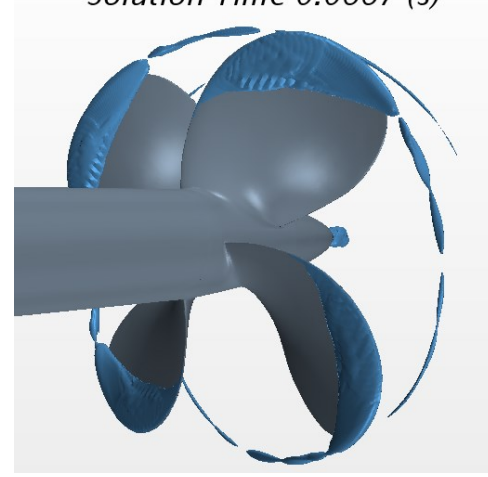

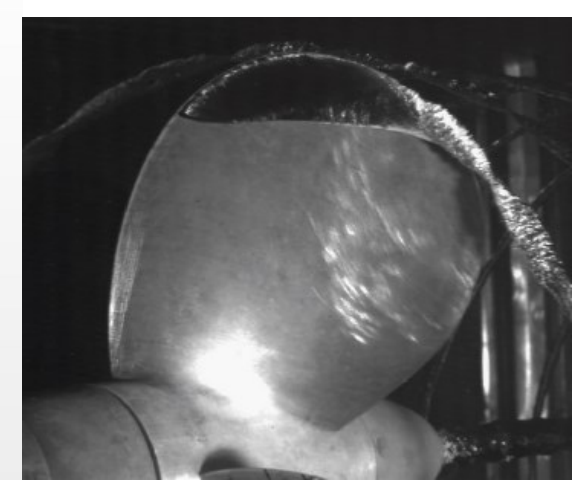

Figure 13. Left; Cavitation pattern using spiral mesh method (Volume fraction of vapour: 0.1), Right; EFD results (Salvatore et al., 2009)

\subsection{Adaptive Mesh Method (Cell Width Method)}

Following improvements with the spiral tip vortex cavitation extension, a new method has been tried to investigate the more efficient generation of the fine spiral mesh. Although the spiral method is useful for extending the tip vortex cavitation in the propeller slipstream, it is not self-adaptive for different operating conditions and propeller geometries. However, the parameters of the spiral geometry -such as pitch, diameter and so on- must be changed for each different rotation speed and advance velocity. Additionally, redundant cells were generated outside the area, where the tip vortex cavitation occurs, due to the changing diameter of the spiral section. Consequently, an "adaptive mesh refinement" method was developed improving the existing solution.

With this new method, the mesh refinement area was created only in the area where the tip vortex cavitation occurs. Before developing this method, the upper limit for absolute pressure was defined by creating a threshold region in STAR-CCM+. The volume fraction of vapour shows the volume, where the absolute pressure is dropping below the saturation pressure of water, hence indicating the location of cavitation volume. When the threshold was created using an absolute pressure value below 10,000 [Pa], which is a higher pressure value than the saturation pressure, a threshold region (the pink area in Figure 14) appeared. This means that if the saturation pressure were accepted as $10,000[\mathrm{~Pa}]$ instead of 3,169 $[\mathrm{Pa}]$, the tip vortex cavitation would be simulated as shown extended in Figure 14. This artifice indicates the volumetric trajectory on which to generate a fine mesh to simulate the pressure drop correctly and capture the cavitation bubbles accurately in that region. 
With this idea, two field functions were created for generating a more refined mesh adapted to extend the tip vortex cavitation within the identified volumetric trajectory of the propeller slipstream.

\section{Field Function 1:}

Cellwidth $=\operatorname{pow}(\$$ Volume, $(1 / 3))$

\section{Field Function 2:}

Refinement $=\$\{$ AbsolutePressure $\}<10000 \& \& \$\{$ AbsolutePressure $\}>3169 ?$

$\$\{$ cellwidth $\} / 3: 0$

The first field function with "Cell Width" defines the one dimension of each cubic mesh cell. The second field function with "Refinement" is defined for creating a refinement table to use while an adaptive mesh is generated. The second field function dictates that each mesh cell, where the absolute pressure below 10000 [Pa] and above 3169 [Pa], will be sub-divided by three in all the three dimensions. Otherwise, the dimension of each cell will be kept the same. The upper limit of absolute pressure was defined by creating a threshold and checking visually as shown in Figure 14. The lowest limit of the pressure is set to the saturation pressure. Because the cavitation has already been captured, in the previous iteration, the region where the absolute pressure is below saturation pressure is known and used to prevent redundant mesh cells being generated. Figure 15 shows the mesh generated using this new adaptive mesh method.

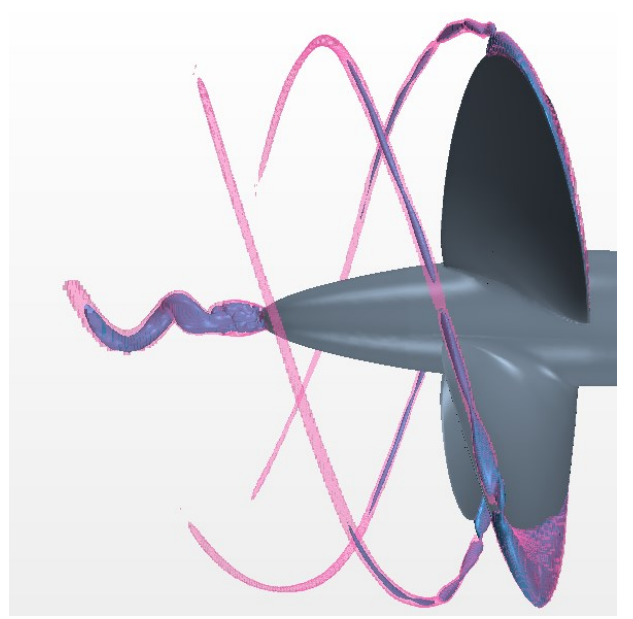

Figure 14. Absolute pressure threshold below 10000 [Pa] 


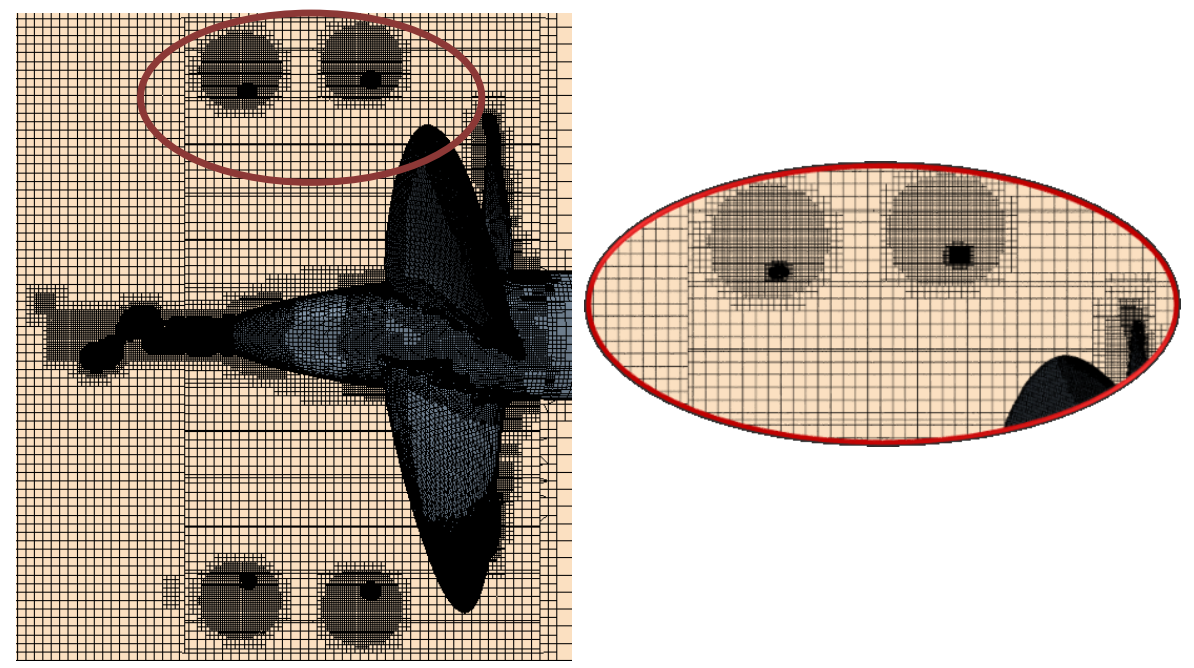

Figure 15. Mesh refinement due to mesh adaption

Before using the adaptive mesh method, the surface size in the spiral geometry was approximately $0.004 \mathrm{D} \mathrm{mm}$. After applying the field functions to create the mesh refinement table, the surface size became $0.001 \mathrm{D} \mathrm{mm}$ by dividing by 3 . The adaptive mesh method gives approximately $0.22 \mathrm{~mm}$ mesh size in the tip vortex cavitation region and a total number of cells of 23 million. Without the new adaptive mesh scheme, the total number of cells would have been more than 100 million and would not have been reasonable for either industrial projects or academic studies due to computational costs and time.

The crucial part of the mesh adaption study is to define the mesh size in the tip vortex region for capturing cavitation bubbles. Insights into cavitation development and cavitation inception have been obtained from measurements carried out by Kuiper (1981), using a model scale propeller (Propeller V) at $\mathrm{J}$ numbers of $0.3,0.4$ and 0.5 including tip vortex cavitation investigations. Kuiper derived an empirical relation between the cavitation index $\left(\sigma_{n}\right)$ and the core radius $\left(a_{c}\right)$ in a series of graphs and equations. The minimum radius of each bubble $\left(a_{i}\right)$ was always measured as about $0.25 \mathrm{~mm}$ at inception. According to these measurements, the core radius $\left(a_{c}\right)$ tends to go to the minimum core radius $\left(a_{i}\right)$. Within the present CFD study, such relations between bubble radius, mesh size and simulating tip vortex cavitation are defined within the new mesh adaption method. The mesh size was kept below $0.25 \mathrm{~mm}$ (it is approximately 0.22 for this simulation provided by dividing existing surface size by 3) for capturing the cavitation in the propeller slipstream. There are roughly $16 \times 12$ cubic cells in the cross-section of the cavitating tip vortex in the finest region. Otherwise, tip vortex cavitation cannot be extended as much as in Figure 16 using a larger mesh size. 

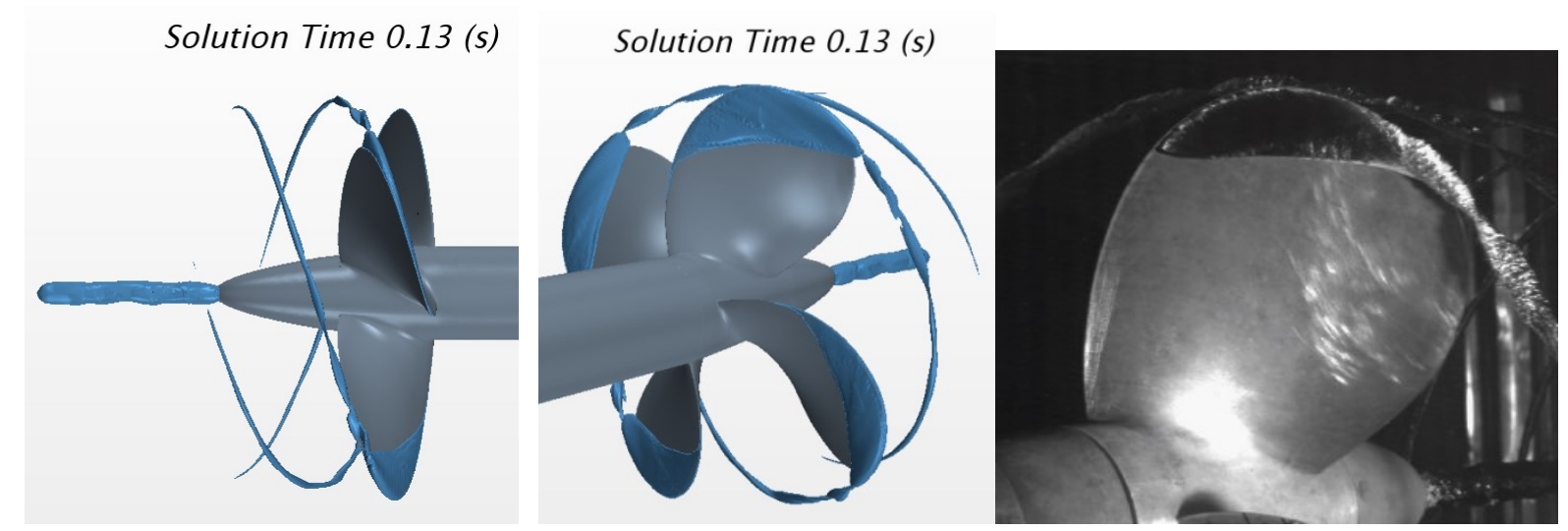

Figure 16. Left; Cavitation pattern using the new adaptive mesh method, Right; EFD results (Salvatore et al, 2009)

\section{A NEW APPROACH TO TIP VORTEX CAVITATION MODELLING - MARCS}

Based on the earlier described new mesh refinement methods in $\S 5$, an overall description of the new cavitation modelling approach, which is named as "Mesh Adaption and Refinement approach for Cavitation Simulation (MARCS)", is given in this section to investigate the interaction between mesh generation and tip vortex cavitation phenomena.

Basically, MARCS presents an enhanced cavitation modelling approach based on an intelligent adaptive meshing technique, and its step-by-step application is defined in the flowchart shown in Figure 15. This approach is used in combination with STAR-CCM+ and applied to simulate tip vortex cavitation trajectories for marine propellers. The tip vortex cavitation extension in the propeller's slipstream can be achieved by following the steps shown in the flowchart. 


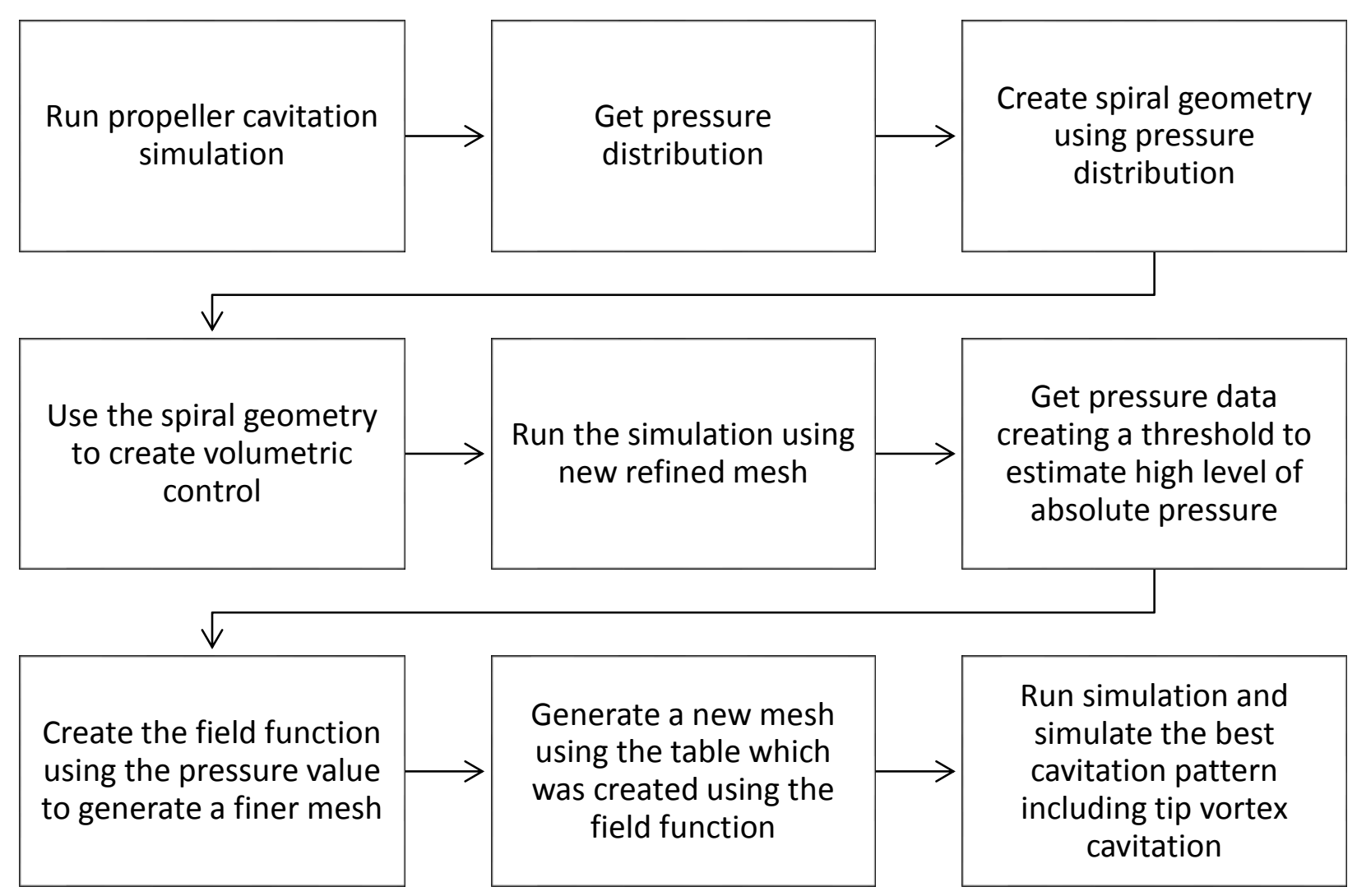

Figure 17. Flowchart summarising new Mesh Adaption and Refinement approach for Cavitation Simulation (MARCS)

\section{RESULTS AND DISCUSSIONS}

At the beginning of this study, the E779A propeller was simulated in non-cavitating conditions. CFD results were compared with experimental results and showed good agreement for a range of propeller performance coefficients.

Following the non-cavitating open water simulations, a cavitating condition of the same simulations was conducted for the INSEAN E779A propeller. In the first simulations, the results showed good agreement with the EFD results for both propeller performance and sheet cavitation patterns on the blade surface. However, tip vortex cavitation could not be simulated. In order to fully simulate the known (EFD) cavitation phenomena (including tip vortex cavitation in the propeller slipstream), a different and advanced mesh refinement method was developed, as outlined in this paper. The preliminary results of this superior method used a tube geometry for capturing particularly tip vortex cavitation in a propeller slipstream and achieved reasonable success in simulating the limited extension of the tip 
vortex cavitation for the INSEAN E779A propeller, as reported by the authors (Yilmaz et al. 2017). In the present paper, the method has been further developed and applied to the same propeller, achieving a greater extension of the cavitating tip vortex in the propeller slipstream. Although the method is based on the mesh refinement of a spiral geometry in tracking the tip vortex, this itself was not efficient to extend the cavitating tip vortex trajectory further in the slipstream. Hence the method was further developed by using computed pressure distributions along the slipstream to track the tip vortex cavitation and apply a newly refined mesh adaption method. This enhanced method helped to extend the tip vortex cavitation until the interface between the rotating and stationary domains in the slipstream. Figure 18 shows the improvement in simulating the tip vortex cavitation extension during this study, thus demonstrating the effectiveness of this new mesh adaption refinement for tip vortex cavitation simulations.

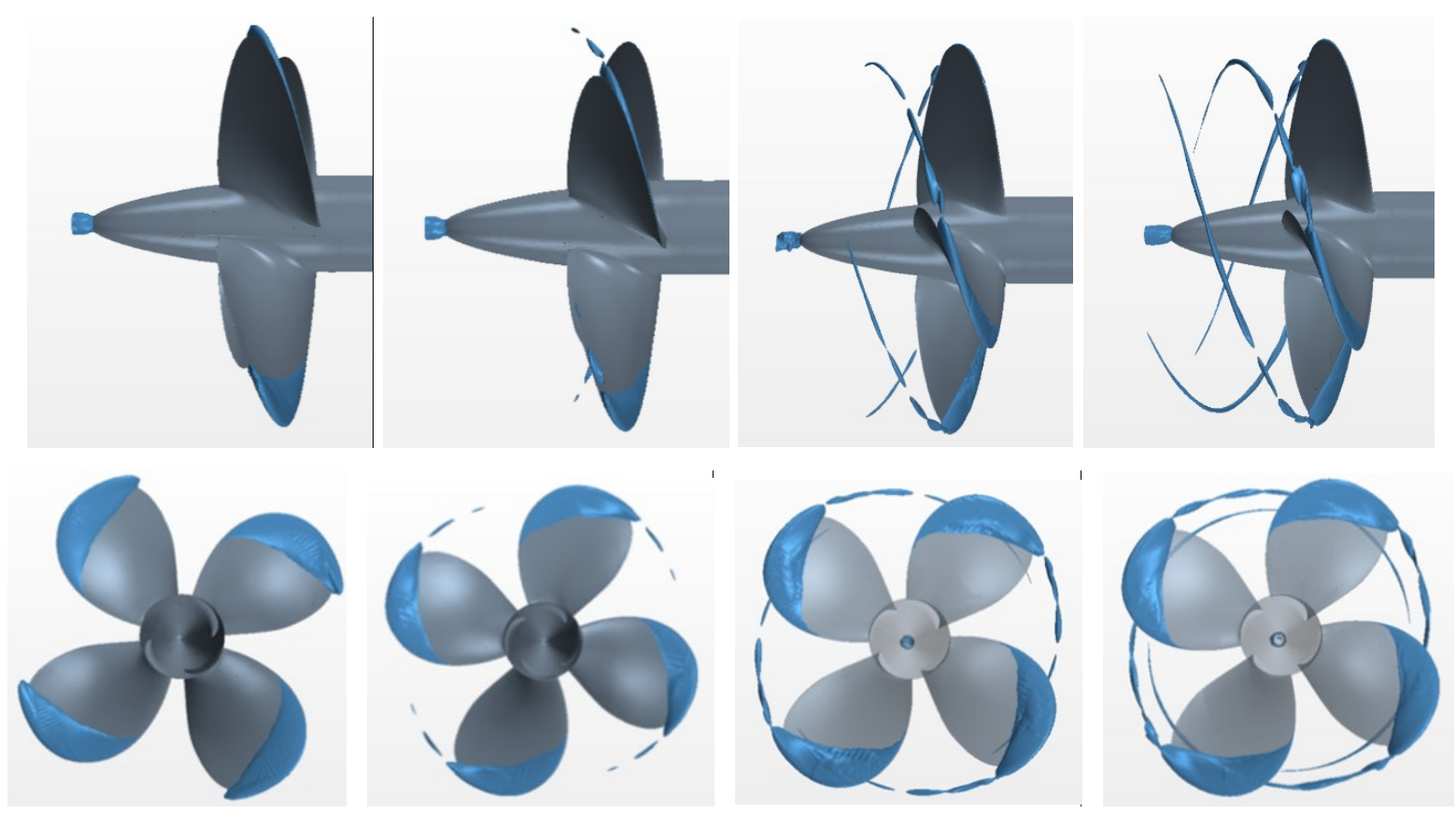

Figure 18. Improvement of tip vortex cavitation extension (From left to right; Result without mesh refinement, with tube refinement, with spiral geometry refinement and with mesh adaption refinement MARCS) 
Table 13. Comparison between CFD and EFD for two mesh methods

\begin{tabular}{|l|c|c|c|c|c|c|c|}
\hline \multicolumn{4}{|c|}{ Performance Coefficient } & \multicolumn{3}{c|}{$\begin{array}{c}\text { Difference } \\
\text { (CFD \& EFD) }\end{array}$} \\
\hline & $\mathrm{J}$ & $\mathrm{K}_{\mathrm{T}}$ & $10 \mathrm{~K}_{\mathrm{Q}}$ & $\eta_{0}$ & $\mathrm{~K}_{\mathrm{T}}$ & $10 \mathrm{~K}_{\mathrm{Q}}$ & $\eta_{0}$ \\
\hline Spiral & 0.71 & 0.244 & 0.439 & 0.627 & $-4 \%$ & $-4 \%$ & $0 \%$ \\
\hline MARCS & 0.71 & 0.246 & 0.443 & 0.629 & $-3 \%$ & $-4 \%$ & $1 \%$ \\
\hline EFD Results & 0.71 & 0.255 & 0.429 & 0.626 & - & - & - \\
\hline
\end{tabular}

Table 13 shows the comparison between results with spiral geometry, mesh adaption refinement (MARCS) and experimental results. The deviation between EFD and CFD results is approximately $4 \%$ for thrust and torque coefficients. Furthermore, the new mesh adaption method showed better results for tip vortex cavitation extension than the spiral geometry method and the thrust coefficient had been calculated closer to the EFD results.
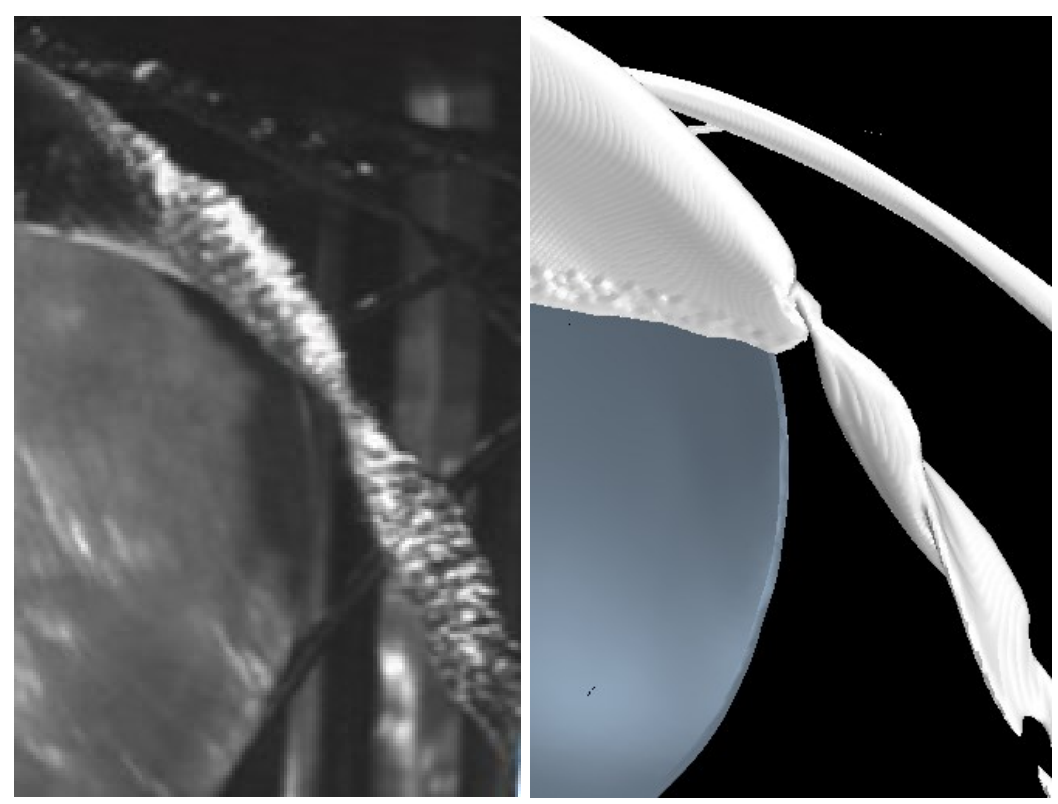

\section{Figure 19. Comparison of Results Tip Vortex Cavitation Roll-up for EFD and CFD}

Finally, Figure 19 shows enlarged comparative images of the cavitating tip vortices, which also include the complex roll-up phenomena in nodes, based on the CFD and EFD. As it can be seen in the EFD picture (on the left of Figure 19), the interaction between the sheet and tip vortex cavitation, which is due to a decrease of the vortex strength or increase of the pressure, resulted in roll-up "nodes" close to the blade trailing edge that have become cylindrical in shape after one pitch downstream of the propeller plane (Kuiper, 1981). As also shown in the 
simulation picture (on the right of Figure 19) this phenomena has been well captured by MARCS.

\section{CONCLUDING REMARKS}

This paper presented a new and efficient Mesh Adaption and Refinement approach to Cavitation Simulation (MARCS) of marine propellers, particularly for the cavitating tip vortex. Simulation of the extension of the cavitating tip vortex well downstream of the propeller plane has been successfully achieved using the new approach as demonstrated with the benchmark INSEAN E779A propeller as well as with other propellers, e.g. Yilmaz et al., (2018).

The MARCS method has been developed using "Absolute Pressure" as the control parameter in the field function in order to be able to refine the mesh in a specific region where the tip vortex cavitation may occur. However, other methods such as vorticity-based approaches can also be used. Some parameters such as the Q-Criterion and Lambda2 will allow vortex identification, are also available in STAR-CCM+ can also be used as parameters in the field function to create the mesh refinement table. These parameters and new field functions will also be investigated to improve the MARCS procedure for tip vortex cavitation simulations in the near future.

Although the MARCS method that has been developed and applied for an isolated propeller, as presented in this paper, the main purpose of this development has been to extend tip vortex cavitation in the propeller slipstream in order to more accurately allow investigations of propeller-rudder and propeller-rudder-hull interactions in the presence of the hull wake from a cavitation point of view. Consequently, this approach is currently being further developed.

\section{ACKNOWLEDGEMENTS}

This paper is based on the Principal author's PhD study which is sponsored by the Turkish Ministry of Education. The access provided to High-Performance Computing for the West of Scotland (ARCHIE-WeSt) thorough EPSRC grant no. EP/K000586/1 is gratefully acknowledged. The authors are also grateful to CRN-INSEAN, especially Francesco Salvatore, for providing the geometry of the E779A propeller and sharing their experimental data. Last but not least, the first author is indebted to Patrick Fitzsimmons for his continuous mentoring and editorial contribution. 


\section{REFERENCES}

Bensow, R., Bark, G., 2010, Simulating Cavitating Flows with Les in Openfoam, V European Conference on Computational Fluid Dynamics, ECCOMAS CFD 2010, June, 2010.

Budich, B., Schmidt, S., J., Adams, N., 2015, Numerical Investigation of a Cavitating Model Propeller Including Compressible Shock Wave Dynamics, Fourth International Symposium on Marine Propulsors smp'15, Austin, Texas, USA, June, 2015.

Fine, N. E. 1992, Nonlinear Analysis of Cavitating Propellers in Nonuniform Flow, PhD thesis, Department of Ocean Engineering, MIT.

Fine, N. E., Kinnas, S, A., 1993, A Boundary Element Method for the Analysis of the Flow Around 3-D Cavitating Hydrofoils, Journal of Ship Research, Vol. 37, No. 3, Sept. 1993, pp. 213-224.

Gaggero, S., Tani, G., Viviani, M. and Conti, F., 2014, A Study on The Numerical Prediction of Propellers Cavitating Tip Vortex, Ocean Engineering 92(2014)137-161.

Guilmineau, E., Deng, G., Leroyer, A., Queutey, P., Visonneau, M., Wackers, J., 2015, Influence of the Turbulence Closures for the Wake Prediction of a Marine Propeller, ERCOFTAC Workshop Direct and Large-Eddy Simulations 10, May 2015, Limassol, Cyprus.

Guilmineau, E., Deng, G., Leroyer, A., Queutey, P., Visonneau, M., Wackers, J., 2015, Influence of the Turbulence Closures for the Wake Prediction of a Marine Propeller, Fourth International Symposium on Marine Propulsors smp'15, Austin, Texas, USA, June 2015.

Hsiao, C. T., Pauley, L. L., 1998, Numerical Computation of Tip Vortex Flow Generated by a Marine Propeller, 1998 ASME Fluids Engineering Division Summer Meeting FEDSM'98 June 21 - 25, 1998 Washington D.C. 
Hsiao, J. T., Chahine, G. L., 2008, Scaling of Tip Vortex Cavitation Inception for a Marine Open Propeller, 27th Symposium on Naval Hydrodynamics Seoul, Korea.

ITTC, 2014, Recommended Procedures and Guidelines, Practical Guidelines for ShipPropulsion CFD, 7.5-03-03-01.

Kinnas, S.A., Fine, N.E., 1994, A Nonlinear Boundary Element Method for the Analysis of Unsteady Propeller Sheet Cavitation, Proceedings of Nineteenth Symposium on Naval Hydrodynamics, Office of Naval Research, National Academy Press, pp. 717-737.

Kinnas, S. A., Hsin, C. Y., 1992, Boundary Element Method for the Analysis of the Unsteady Flow Around Extreme Propeller Geometries, AIAA Journal Vol. 30, No. 3.

Kuiper G., 1981, Cavitation Inception on Ship Propeller Models, Wageningen.

Lee, C. S., 1979, Prediction of Steady and Unsteady Performance of Marine Propellers with or without Cavitation by Numerical Lifting-Surface Theory, PhD Dissertation, Massachusetts Institute of Technology.

Lee, H., 2002, Modeling of Unsteady Wake Alignment and Developed Tip Vortex Cavitation, PhD Dissertation, University of Texas at Austin.

Lee, H., Kinnas, S. A., 2004, Application of a Boundary Element Method in the Prediction of Unsteady Blade Sheet and Developed Tip Vortex Cavitation on Marine Propellers, Journal of Ship Research, Vol. 48, No. 1, March 2004, pp. 15-30.

Lloyd, T., Vaz, G., Rijpkema, D., Reverberi, A., 2017, Computational fluid dynamics prediction of marine propeller cavitation including solution verification, Fifth International Symposium on Marine Propulsors smp'17, Espoo, June 2017

Morgut, M., Nobile, E., 2012, Numerical Predictions of Cavitating Flow around model Scale Propellers by CFD and Advanced Model Calibration, Hindawi Publishing Corporation International Journal of Rotating Machinery Volume 2012, Article ID 
Muzaferija, S., Papoulias, D., Peric, M., 2017, VOF Simulations of Hydrodynamic Cavitation Using the Asymptotic and Classical Rayleigh-Plesset Models, Fifth International Symposium on Marine Propulsion smp'17, Espoo, Finland, June 2017.

Pereira, F., Felice, F. D., Salvatore, F., 2016, Propeller Cavitation in Non-Uniform Flow and Correlation with the Near Pressure Field, Journal of Marine Science and Engineering, 4, 70; doi:10.3390/jmse4040070

Pereira, F., Salvatore, F., Felice, F. D., 2004, Measurement and Modeling of Propeller Cavitation in Uniform Inflow, Journal of Fluids Engineering, Vol. 126.

Peric, M., 2002, Computational Methods for Fluid Dynamics, Springer, Third, Rev. Edition

Roache, P. J., 1998, Verification and Validation in Computational Science and Engineering, Hermosa Publishers, Albuquerque, New Mexico.

Salvatore, F., Streckwall, H., Terwisga, T. 2009, Propeller Cavitation Modelling by CFDResults from the VIRTUE 2008 Rome Workshop, First International Symposium on Marine Propulsors smp'09, Trondheim, Norway.

Schnerr, G. H., Sauer, J., 2001, Physical and Numerical Modeling of Unsteady Cavitation Dynamics, ICMF-2001, $4^{\text {th }}$ International Conference on Multiphase Flow, New Orleans, USA, May 27-June 1, 2001

STAR-CCM+ User Guide, 2018

Stern, F., Wilson, R.V., Coleman, H. W., Paterson, E.R, 2001a, Verification and Validation of CFD Simulations - Part1: Methodology and Procedures, Journal of Fluids Engineering, Vol. 123. 
Vaz, G., Bosschers, J., Modelling, 2006, Three Dimensional Sheet Cavitation on Marine Propellers Using a Boundary Element Method, Sixth International Symposium on Cavitation CAV2006, Wageningen, The Netherlands

Vaz, G., Hally, D., Huuva, T., Bulten, N., Muller, P., Becchi, P., Herrer, J., Whitworth S., Mace, R., Korsström, A., 2015, Cavitating Flow Calculations for the E779A Propeller in Open Water and Behind Conditions: Code Comparison and Solution Validation, Fourth International Symposium on Marine Propulsors smp'15, Austin, Texas, USA.

Viitanen, V, M, Siikonen, T., 2017, Numerical Simulation of Cavitating Marine Propeller Flows, $9^{\text {th }}$ National Conference on Computational Mechanics, MekIT'17.

Wilson, R.V., Stern, F., Coleman, H. W., Paterson, E.R, 2001b, Verification and Validation of CFD Simulations - Part2: Application for RANS simulation of a Cargo/Container Ship, Journal of Fluids Engineering, Vol. 123.

Windt, J., Bosschers, J., 2015, Influence of Local and Adaptive Mesh Refinement on the Tip Vortex Characteristics of a Wing and Propeller, VI International Conference on Computational Methods in Marine Engineering, (MARINE 2015).

Yilmaz, N., Khorasanchi, M., Atlar, M., 2017, An Investigation into Computational Modelling of Cavitation in a Propeller's Slipstream, Fifth International Symposium on Marine Propulsion smp'17, Espoo, Finland, June 2017

Yilmaz, N., Atlar, M., Fitzsimmons, A., P., 2018, An Improved Tip Vortex Cavitation Model for Propeller-Rudder Interaction, The 10th International Symposium on Cavitation (CAV2018), Baltimore, Maryland, USA, 14-16 May 2018.

Young, Y.L., Kinnas S, A., 2001, A BEM for the Prediction of Unsteady Midchord Face and/or Back Propeller Cavitation, J. Fluids Eng 123(2), 311-319, Jan. 